Notions of s/comp in Topological Algebras over the Reals 


\title{
Notions of Semicomputability in Topological Algebras over the Reals
}

By

Mark Armstrong, B.ASc.

\author{
A Thesis \\ Submitted to the School of Graduate Studies \\ in partial fulfilment of the requirements for the degree of \\ Master of Science \\ Department of Computing and Software \\ McMaster University \\ (C) Copyright by Mark Armstrong, August 2015
}


M.Sc. Thesis - M. Armstrong; McMaster University - Computer Science

MASTER OF SCIENCE (2015)

(Computing and Software)

McMaster University

Hamilton, Ontario

TITLE: Notions of Semicomputability in Topological Algebras over the Reals

AUTHOR: Mark Armstrong, B.ASc.(McMaster University)

SUPERVISOR: Prof. Jeffery I. Zucker

NUMBER OF PAGES: viii, 60 


\section{Lay Abstract}

We investigate to what extent certain well-known results of classical computability theory on the natural numbers hold in the context of generalised computability theories on the real numbers. 


\section{Abstract}

Several results from classical computability theory (computability over discrete structures such as the natural numbers and strings over finite alphabets, due to Turing, Church, Kleene and others) have been shown to hold for a generalisation of computability theory over total abstract algebras, using for instance the model of While computation.

We present a number of results relating to computation on topological partial algebras, again using While computation. We consider several results from the classical theory in the context of topological algebra of the reals: closure of semicomputable sets under finite union (Chapter 4 Theorem 2, p.33), the equivalence of semicomputable and projectively (semi)computable sets (Chapter 5 Theorem 7, p.45), and Post's Theorem (i.e. a set is computable iff both it and its complement are semicomputable) (Appendix B Theorem 9, p.53).

This research has significance in the field of scientific computation, which is underpinned by computability on the real numbers. We will consider a "continuity principle", which states that computability should imply continuity; however, equality, order, and other total boolean-valued functions on the reals are clearly discontinuous. As we want these functions to be basic for the algebras under consideration, we resolve this incompatibility by redefining such functions to be partial, leading us to consider topological partial algebras. 


\section{Acknowledgements}

I would like to earnestly thank my supervisor, Dr. Jeffery Zucker, for his tutelage throughout my Masters studies. His insights during my research and his guidance during writing made my thesis possible.

I also deeply thank Dr. Ming Quan Fu, whose Ph.D. thesis contained the basis for my Strucure Theorem. It was also of much guidance when putting together my introduction and background chapters. I came to know Ming Quan as Dr. Zucker's other computer science graduate student, and he also offered much advise and encouragement through my studies.

Many thanks to Professor Bröcker of Münster University for his input on the properties of semi-algebraic and basic sets.

Thanks to the members of my M.Sc. committee, Dr. Wolfram Kahl and Dr. Sanzheng Qiao for their careful and thorough review of the draft of my thesis and for the valuable comments they provided.

Thanks to my dear wife, Sarah, who together with our daughter Callie is the light of my life and the source of my joy. Thanks also to my parents, and to my brother and my sister-in-law, for all their support throughout my studies. 


\section{Contents}

Lay Abstract ........................ iii

Abstract ........................ iii

Acknowledgements ................... iv

1 Introduction 1

1.1 Generalising computability theory . . . . . . . . . . 1

1.2 Overview of the thesis $\ldots \ldots \ldots \ldots \ldots$

2 Signatures; Algebras; the While language 4

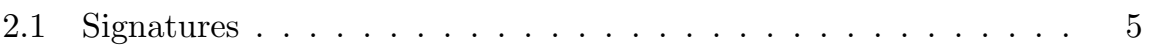

2.2 Algebras . . . . . . . . . . . . . . . 6

2.3 Relations and projections . . . . . . . . . . . . . . 9

2.4 Topological partial algebras . . . . . . . . . . . . . . . 9

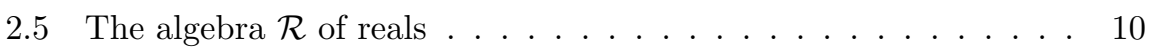

2.6 The algebra $\mathcal{R}^{*} \ldots \ldots \ldots \ldots \ldots \ldots \ldots$

2.7 The While programming language . . . . . . . . . . 13

2.8 States . . . . . . . . . . . . . . . . . . . . . . . . 15

2.9 Semantics of the While language . . . . . . . . . 16

2.10 While computability and semicomputability . . . . . . . 18

2.11 Extending While to While $\mathrm{OR}^{\mathrm{R}}$ and While $^{\exists \mathrm{N}} \ldots \ldots \ldots$ 
2.12 Extending While, While ${ }^{\mathrm{OR}}$ and $\boldsymbol{W h i l e}^{\exists \mathrm{N}}$ to their starred versions 20

2.13 Encoding of syntax . . . . . . . . . . . . . . 20

3 Semantic disjointedness; Engeler's Lemma 22

3.1 Engeler's Lemma . . . . . . . . . . . . . . . . 22

3.2 Canonical form for booleans over $\mathcal{R}$. . . . . . . . . . . . . . 23

3.3 Semi-algebraic and basic sets . . . . . . . . . . . 23

3.4 Positive and negative sets . . . . . . . . . . . . . . . . . 24

4 While $(\mathcal{R})$ semicomputable sets: Structure Theorem and failure $\begin{array}{ll}\text { of closure under union } & 26\end{array}$

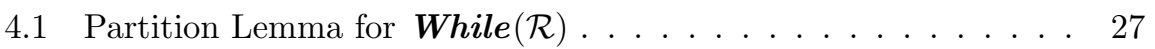

4.2 Structure Theorem for $\boldsymbol{W h i l e}(\mathcal{R})$ semicomputability . . . . . . . 32

4.3 Failure of closure of While $(\mathcal{R})$ semicomputable sets under union 33

5 Classes of sets semicomputable by models based on the While $\begin{array}{ll}\text { language } & 37\end{array}$

5.1 A set which is projectively $\operatorname{While}(\mathcal{R})$ semicomputable but not While $(\mathcal{R})$ semicomputable . . . . . . . . . . . . . . . 37

5.2 A set which is projectively While $^{\mathrm{OR}}(\mathcal{R})$ semicomputable but not While ${ }^{\mathrm{OR}}$ semicomputable ............... 40

5.3 Equivalence of projective $\boldsymbol{W h i l e}(\mathcal{R})$ and $\boldsymbol{W h i l e}^{\exists \mathrm{N}}(\mathcal{R})$ semicomputability .............................. 42

5.4 Classes of sets semicomputable by models based on the While language ....................... 44

6 Conclusion and future work 46

6.1 Conclusion .................... 46

6.2 Future work . . . . . . . . . . . . . . . . 47 
A The equivalence of While $(\mathcal{R})$ and While $^{*}(\mathcal{R})$ 


\section{Chapter 1}

\section{Introduction}

\subsection{Generalising computability theory}

In classical computability theory, many formalisms have been presented and been proven to be equivalent, including the formalism of Turing machines, $\lambda$-calculus, and the $\mu$-recursive functions, presented by Alan Turing [Tur36], Alonzo Church [Chu36] and Stephen Kleene [Kle36] respectively during the 1930's. These all capture the informal notion of computation by a finite, deterministic algorithm on $\mathbb{N}$ or on $\Sigma^{*}$ (the set of strings from a finite alphabet $\Sigma$ ).

We generalise the classical computability theory to other abstract structures, especially the domain of real numbers $\mathbb{R}$.

Our reason for generalising to abstract models is that scientific computation is done largely on the reals, so we wish to apply the techniques of classical computability theory to the set $\mathbb{R}$ of real numbers and similar sets.

An important difference between $\mathbb{R}$ and $\mathbb{N}$ is that real numbers can only be constructed as infinite objects, for instance, as infinite sequences of rational numbers. Thus when working with $\mathbb{R}$, at least with concrete computation mod- 
els (described below), we must work with the ideas of finite approximations. Further the topology of the reals gives us the idea of "nearness", and the closeness of approximations. We will see that the topology of the reals is a crucial concept in computation over the reals.

A model of computation is a mathematical model of some general method of computing functions, or deciding membership of a set. We distinguish two main kinds of such models: abstract and concrete.

For abstract models of computation, the data are taken as primitives, so the programs and algorithms do not depend on representations.

Examples of abstract models of computation are high level programming language, flow charts and register machines over any algebra.

For concrete models data are given by representations, and so the programs and algorithms are highly dependent on the choice of representation. For example, the reals may have finite representations by (indices of) effective Cauchy sequences.

Examples of concrete models of computation are "tracking computability" [TZ04, TZ05], Grzegorczyk-Lacombe computability [Grz55, Grz57] and Weihrauch's Type 2 computability [Wei00].

An important part of our work in this thesis is to consider whether certain results from the classical theory still hold in the generalised computability theory. We also show the equivalence and inequivalence of certain abstract models of computation based on the While language over $\mathbb{R}$.

\subsection{Overview of the thesis}

In Chapter 2 we review many-sorted structures and algebras, relations and projections, topological partial algebras (in particular the algebra $\mathcal{R}$ on $\mathbb{R}$ with the ring structure of the reals), and the While, While ${ }^{\mathrm{OR}}$ and While $\boldsymbol{W N}^{\mathrm{N}}$ program- 
ming languages over $\mathcal{R}$, as well as the projective and "starred" versions of those languages.

In Chapter 3 we give lemmas and definitions for the While language on $\mathcal{R}$ that we use to present a Structure Theorem for While $(\mathcal{R})$ in Chapter 4. Using that Structure Theorem, we prove that the set of While $(\mathcal{R})$ semicomputable sets is not closed under union.

In Chapter 5 we present results regarding the equivalence of models of computation on $\mathcal{R}$ based on the While language.

In Chapter 6 we present our conclusion, and some ideas for about future work.

In Appendix A we prove the equivalence of the starred versions of models based on the While language.

In Appendix B we show that another closure result from the classical theory, Post's Theorem, holds trivially in the case of $\mathcal{R}$, but does not hold more generally for partial algebras. 


\section{Chapter 2}

\section{Signatures; Algebras; the}

\section{While language}

We will study the computation of functions and relations by high level imperative programming languages based on the 'while' construct, applied to a many-sorted signature $\Sigma$. We give semantics for this language relative to a topological partial $\Sigma$-algebra $A$, and define the notions of computability, semicomputability and projective semicomputability for this language on A. Much of the material is taken from [TZ00, TZ15], adapted to partial algebras.

We begin by reviewing basic concepts: many-sorted signatures and algebras. Next we define the syntax and semantics of the While programming language. Then we present several extensions to this language. 


\subsection{Signatures}

Definition 2.1.1 (Signature). A many-sorted signature $\Sigma$ is a pair $\langle\boldsymbol{S o r t}(\Sigma), \boldsymbol{F u n c}(\Sigma)\rangle$ where

(a) $\operatorname{Sort}(\Sigma)$ is a finite set of basic types called sorts $s, s^{\prime}, \ldots$.

(b) Func $(\Sigma)$ is a finite set of basic function symbols

$$
F: s_{1} \times \cdots \times s_{m} \rightarrow s \quad(m \geq 0)
$$

The case $m=0$ gives a constant symbol; we then write $F: \rightarrow s$.

Definition 2.1.2 (Product Type). A product type of $A$ has the form $s_{1} \times \cdots \times s_{m}$, where $m \geq 0$ and $s_{1}, \ldots, s_{m}$ are sorts of $A$. We write $u, v, \ldots$ for product types. A function type has the form $u \rightarrow s$, where $u$ is a product type.

Definition 2.1.3 (Standard Signature). A signature is called standard if it includes the sorts and functions of the signature of the booleans:

\begin{tabular}{|ll|}
\hline signature & $\Sigma(\mathcal{B})$ \\
sorts & bool \\
functions & true, false : $\rightarrow$ bool \\
& or, and $:$ bool ${ }^{2} \rightarrow$ bool \\
& cor, cand $:$ bool ${ }^{2} \rightarrow$ bool \\
& not $:$ bool $\rightarrow$ bool
\end{tabular}

All signatures used in this thesis are standard. 
Definition 2.1.4 ( $\mathbb{N}$-standard Signature). A signature is called $\mathbb{N}$-standard if, in addition to being standard, it includes the sorts and functions of the signature of the naturals:

\begin{tabular}{|ll|}
\hline signature & $\Sigma(\mathcal{N})$ \\
sorts & nat \\
functions & $0: \rightarrow$ nat \\
& suc $:$ nat $\rightarrow$ nat \\
& eq, less $:$ nat $^{2} \rightarrow \mathbb{B}$ \\
\hline
\end{tabular}

All signatures used in our main results are $\mathbb{N}$-standard ${ }^{1}$.

\section{$2.2 \quad$ Algebras}

Definition 2.2.1 (Algebra). For a signature $\Sigma$, a $\Sigma$-algebra $A$ has, for each sort $s$ of $\Sigma$, a non-empty set $A_{s}$, called the carrier of sort $s$, and for each function symbol $F: s_{1} \times \ldots \times s_{m} \rightarrow s$, a function $F^{A}: A_{s_{1}} \times \ldots \times A_{s_{1}} \rightarrow A_{s}$.

Notation 2.2.2. We write $\Sigma(A)$ for the signature of an algebra $A$.

Notation 2.2.3. For a $\Sigma$-product type $u=s_{1} \times \ldots \times s_{m}$, we write

$$
A^{u}={ }_{d f} A_{s_{1}} \times \ldots \times A_{s_{m}}
$$

Definition 2.2.4 (Total and Partial Algebras). An algebra is total if $f^{A}$ is total for all $f \in \boldsymbol{F u n c}(\Sigma)$; otherwise, it is partial.

\footnotetext{
${ }^{1}$ The signature used in Appendix B is not $\mathbb{N}$-standard, for the sake of the simplicity of the proof in that chapter.
} 
The (total) algebras of the booleans and the naturals are as follows:

$\begin{array}{ll}\text { algebra } & \mathcal{B} \\ \text { carrier } & \mathbb{B} \\ \text { functions } & \text { true }^{\mathrm{B}}, \text { false }^{\mathrm{B}}: \rightarrow \mathbb{B} \\ & \text { or }^{\mathrm{B}}, \text { and }^{\mathrm{B}}: \mathbb{B}^{2} \rightarrow \mathbb{B} \\ & \operatorname{cor}^{\mathrm{B}}, \text { cand }^{\mathrm{B}}: \mathbb{B}^{2} \rightarrow \mathbb{B} \\ & \operatorname{not}^{\mathrm{B}}: \mathbb{B} \rightarrow \mathbb{B}\end{array}$

\begin{tabular}{|ll|}
\hline algebra & $\mathcal{N}$ \\
carriers & $\mathbb{N}$ \\
functions & $0^{\mathrm{N}}: \rightarrow \mathbb{N}$ \\
& $\operatorname{suc}^{\mathrm{N}}: \mathbb{N} \rightarrow \mathbb{N}$ \\
& eq $^{\mathrm{N}}$, less $^{\mathrm{N}}: \mathbb{N}^{2} \rightarrow \mathbb{B}$
\end{tabular}

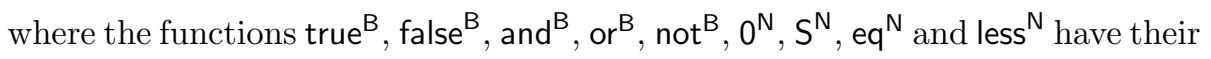
usual definitions, and the functions $\operatorname{cor}^{\mathrm{B}}$ and $\operatorname{cand}^{\mathrm{B}}$ are defined as in Definition 2.2.5.

We will often write $\vee$ and $\wedge$ in place of or and and, $\stackrel{c}{\vee}$ and $\stackrel{c}{\wedge}$ in place of cor and cand and $\neg$ in place of not, and drop the superscripts ${ }^{B}$ and ${ }^{N}{ }^{N}$ where unambiguous. 
Definition 2.2.5. The semantics of the "conditional operators" $\stackrel{c}{\vee}^{c}$ and $\stackrel{c}{\wedge}$ are as follows:

\begin{tabular}{|c|c|c|c|c|}
\hline \multirow{4}{*}{$\llbracket b_{1} \stackrel{c}{\vee}^{b} b_{2} \rrbracket^{\mathcal{B}} \sigma$} & $\llbracket b_{1} \rrbracket^{\mathcal{B}} \sigma \quad \llbracket b_{2} \rrbracket^{\mathcal{B}} \sigma$ & true $^{B}$ & false ${ }^{B}$ & $\uparrow$ \\
\hline & true $^{\mathrm{B}}$ & true $^{\mathrm{B}}$ & true $^{\mathrm{B}}$ & true $^{\mathrm{B}}$ \\
\hline & false ${ }^{B}$ & true $^{B}$ & false ${ }^{B}$ & $\uparrow$ \\
\hline & $\uparrow$ & $\uparrow$ & $\uparrow$ & $\uparrow$ \\
\hline
\end{tabular}

\begin{tabular}{|c|c|c|c|c|}
\hline \multirow{4}{*}{$\llbracket b_{1} \stackrel{c}{\wedge} b_{2} \rrbracket^{\mathcal{B}} \sigma$} & $\llbracket b_{1} \rrbracket^{\mathcal{B}} \sigma \llbracket \llbracket b_{2} \rrbracket^{\mathcal{B}} \sigma$ & $\operatorname{true}^{\mathrm{B}}$ & false ${ }^{B}$ & $\uparrow$ \\
\hline & $\operatorname{true}^{\mathrm{B}}$ & true $^{B}$ & false ${ }^{B}$ & $\uparrow$ \\
\hline & false ${ }^{B}$ & false $^{B}$ & false $e^{B}$ & false ${ }^{B}$ \\
\hline & $\uparrow$ & $\uparrow$ & $\uparrow$ & $\uparrow$ \\
\hline
\end{tabular}

i.e., the operators $\stackrel{c}{\vee}$ and $\stackrel{c}{\wedge}$ are "evaluated from the left".

The definition of $\llbracket \cdot \rrbracket^{\mathcal{B}} \sigma$ is given in Section 2.8 .

Definition 2.2.6 (Standard Algebra). An algebra is called standard if it is an expansion of $\mathcal{B}$ and any equality operators, for values on which they are defined, they are the identity of their respective sorts.

All algebras in this thesis are standard.

Definition 2.2.7 (N-standard Algebra). An algebra is called $\mathbb{N}$-standard if it is an expansion of $\mathcal{N}$.

All algebras used in our main results are $\mathbb{N}$-standard ${ }^{3}$.

${ }^{2}$ The operators $\stackrel{c}{\vee}$ and $\stackrel{c}{\wedge}$ are called conditional operators because they can be simulated using conditional statements. Thus, in the While language, $b_{1} \stackrel{c}{\vee} b_{2}$ can be simulated by "if $b_{1}$ then true else $b_{2}$ fi" and $b_{1} \stackrel{c}{\wedge} b_{2}$ by "if $\neg b_{1}$ then false else $b_{2}$ fi".

${ }^{3}$ See footnote 1 . 


\subsection{Relations and projections}

Let $\Sigma$ be any signature and $A$ any $\Sigma$-algebra.

Definition 2.3.1 (Relation). A relation on $A$ of type $u$ is a subset of $A^{u}$. We write $R: u$ if $R$ is a relation of type $u$.

Definition 2.3.2 (Complement of a Relation). The complement of $R$ in $A$ is the relation

$$
R^{c}=A^{u} \backslash R=\left\{a \in A^{u} \mid a \notin R\right\}
$$

Definition 2.3.3 (Projection). Let $R$ be a relation of type $u=s_{1} \times \ldots \times s_{m}$ where $m>0$. Let $\vec{i}=i_{1}, \ldots, i_{r}$ be a list of numbers such that $1 \leq i_{1}<\ldots<i_{r} \leq m$, and let $\vec{j}=j_{1}, \ldots, j_{m-r}$ be the list $\{1, \ldots, m\} \backslash \vec{i}$. Then the projection of $R$ off of $i$ is the relation $S: s_{j_{1}} \times \ldots \times s_{j_{m-r}}$ such that:

$$
S\left(x_{j_{1}}, \ldots, x_{j_{m-r}}\right) \Longleftrightarrow \exists x_{i_{1}}, \ldots, x_{i_{r}}: s_{i_{1}}, \ldots, s_{i_{r}}, R\left(x_{1}, \ldots, x_{m}\right)
$$

\subsection{Topological partial algebras}

Recall the definition of continuity of partial functions ${ }^{4}$ :

Definition 2.4.1 (Continuity). Given two topological spaces $X$ and $Y$, a partial function $f: X \rightarrow Y$ is continuous if for every open $V \subseteq Y$,

$$
f^{-1}[V]={ }_{d f}\{x \in X \mid x \in \boldsymbol{d o m}(f) \text { and } f(x) \in V\}
$$

is open in $X$.

Definition 2.4.2 (Topological partial algebra). A topological partial algebra is a partial $\Sigma$-algebra with topologies on the carriers such that each of the basic $\Sigma$-functions is continuous. The carriers $\mathbb{B}$ and $\mathbb{N}$, if present, have the discrete topology.

\footnotetext{
${ }^{4}$ For more information on topologies, topological spaces and continuity, refer to any standard topology text, such as Rudin's Principles of Mathematical Analysis.
} 
Remark 2.4.3 (Continuity of computable functions; the continuity principle). The significance of the continuity of the basic functions of a topological algebra $A$ is that it implies continuity of all While computable function on $A$ [TZ99, TZ00].

This is in accordance with the Continuity Principle which can be expressed as

$$
\text { computability } \Longrightarrow \text { continuity. }
$$

This principal is discussed in [TZ99, TZ04].

\subsection{The algebra $\mathcal{R}$ of reals}

In the following sections, we work mostly with the following topological algebra ${ }^{5}$ :

$$
\begin{aligned}
& \text { algebra } \mathcal{R} \\
& \text { carriers } \quad \mathbb{R}, \mathbb{B}, \mathbb{N} \\
& \text { functions } 0^{\mathrm{R}}, 1^{\mathrm{R}}: \rightarrow \mathbb{R} \\
& \text { plus }^{\mathrm{R}} \text {, times }{ }^{\mathrm{R}}: \mathbb{R}^{2} \rightarrow \mathbb{R} \\
& 0^{\mathrm{N}}: \rightarrow \mathbb{N} \\
& \operatorname{suc}^{\mathrm{N}}: \mathbb{N} \rightarrow \mathbb{N} \\
& \text { true, false : } \rightarrow \mathbb{B} \\
& \text { or, and }: \mathbb{B}^{2} \rightarrow \mathbb{B} \\
& \text { cor, cand : } \mathbb{B}^{2} \rightarrow \mathbb{B} \\
& \text { not }: \mathbb{B} \rightarrow \mathbb{B} \\
& \text { eq }^{\mathrm{N}}, \text { less }^{\mathrm{N}}: \mathbb{N}^{2} \rightarrow \mathbb{B} \\
& \text { eq }^{\mathrm{R}}, \text { less }^{\mathrm{R}}: \mathbb{R}^{2} \rightarrow \mathbb{B}
\end{aligned}
$$

\footnotetext{
${ }^{5}$ In [Fu14], this algebra was called $\mathcal{R}_{0} ; \mathcal{R}$ was the algebra which also included the inverse operation for the reals.
} 


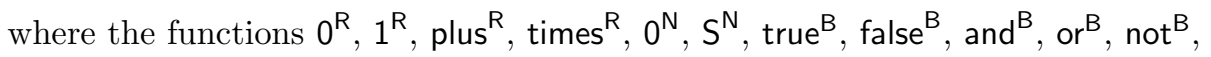
$\mathrm{eq}^{\mathrm{N}}$ and less ${ }^{\mathrm{N}}$ have their usual definitions, the functions cor and cand are defined as in Definition 2.2.5, and the function eq ${ }^{\mathrm{R}}$ and less $^{\mathrm{R}}$ are defined as in Remark 2.5.1.

We will often write $=$ and $<$ in place of eq $^{\mathrm{R}}$ and less ${ }^{\mathrm{R}}$, and drop the superscripts $\cdot{ }^{R}, \cdot{ }^{B}$ and $\cdot N$ where unambiguous

The signature $\Sigma(\mathcal{R})$ can be inferred from the above, with real as the sort of $\mathbb{R}$.

Remark 2.5.1. $\mathcal{R}$ is a partial algebra, with the basic partial functions eq ${ }^{\mathrm{R}}$ and less $^{\mathrm{R}}$, where for $x, y \in \mathbb{R}$ :

$$
\llbracket \mathrm{eq}^{\mathrm{R}}(x, y) \rrbracket^{\mathcal{R}} \sigma \simeq \begin{cases}\uparrow & \text { if } \llbracket x \rrbracket^{\mathcal{R}} \sigma=\llbracket y \rrbracket^{\mathcal{R}} \sigma \\ \text { false } & \mathrm{o} / \mathrm{w}\end{cases}
$$

and

$$
\llbracket \text { less }^{\mathrm{R}}(x, y) \rrbracket^{\mathcal{R}} \sigma \simeq \begin{cases}\text { true } & \text { if } \llbracket x \rrbracket^{\mathcal{R}} \sigma<\llbracket y \rrbracket^{\mathcal{R}} \sigma \\ \text { false } & \text { if } \llbracket x \rrbracket^{\mathcal{R}} \sigma>\llbracket y \rrbracket^{\mathcal{R}} \sigma \\ \uparrow & \text { o/w. }\end{cases}
$$

The definition of $\llbracket \cdot \rrbracket^{\mathcal{R}} \sigma$ is given in section 2.8 .

By contrast, the basic functions eq ${ }^{\mathrm{N}}$ and less ${ }^{\mathrm{N}}$ on $\mathbb{N}$, are total.

Notation 2.5.2. The symbol ' $\simeq$ ' denotes Kleene equality, where the two sides are either both defined and equal, or both undefined.

Discussion 2.5.3 (Motivation for use of partial functions). We present our motivation for using partial functions in two ways. The first is a discussion of continuity of comparison operators on $\mathbb{R}$. The second is a Gedankenexperiment involving concrete models of computation. 
(1) The total versions of the comparison operators eq ${ }^{\mathrm{R}}$ and less ${ }^{\mathrm{R}}$ on $\mathbb{R}$ are not continuous. (By contrast any comparison operators on $\mathbb{N}$ are continuous, because $\mathbb{N}$ has the discrete topology). Continuity of these comparison operators is important due to the Continuity Principle (Remark 2.4.3) and our definition of topological partial algebras (Definition 2.4.2), which requires all basic operators to be continuous.

(2) Consider now the task of determining whether for two real variables $\mathrm{x}$ and $\mathrm{y}, \mathrm{x}=\mathrm{y}$, in some concrete model of computation. For this example, let us use as a representation of these real numbers effective Cauchy sequences of rationals $\left(r_{0}, r_{1}, r_{2}, \ldots\right)$ and $\left(s_{0}, s_{1}, s_{2}, \ldots\right)$. We assume for our convenience that the sequences are "fast", i.e.,

$$
\forall n, \forall m \geq n,\left|r_{n}-r_{m}\right|<2^{-n}
$$

and similarly for $\left(s_{n}\right)$. Now suppose that for $n=1,2,3, \ldots$ the inputs $r_{n}$ and $s_{n}$ are observed (from some device) at $n$ time units. Now $\mathrm{x}<\mathrm{y}$ is true at $\sigma$ iff for some $n, r_{n}+2 \cdot 2^{-n}<s_{n}$, and this can be determined in a finite amount of time. Correspondingly, $\mathrm{x}=\mathrm{y}$ is true iff for all $n$, $\left|r_{n}-s_{n}\right|<2 \cdot 2^{-n}$, but this cannot be determined in a finite amount of time. So from this example it is natural for comparison operators on $\mathrm{x}$ and $\mathrm{y}$ to diverge in cases when $\mathrm{x}=\mathrm{y}$.

Remark 2.5.4. Throughout the main results of our thesis we focus on functions on $\mathbb{R}^{2}$. There are many problems to consider on $\mathbb{R}^{2}$ that are not present for functions on $\mathbb{R}$; for instance, While semicomputable sets on $\mathbb{R}$ are closed under union $^{6}$.

\footnotetext{
${ }^{6}$ This is clear from the characterization of sets on $\mathbb{R}$ given in [XFZ15].
} 


\subsection{The algebra $\mathcal{R}^{*}$}

The algebra $\mathcal{R}^{*}$ is formed from $\mathcal{R}$ by adding the carriers $\mathbb{R}^{*}, \mathbb{N}^{*}$ and $\mathbb{B}^{*}$ (of sorts real*, nat* and bool* respectively) consisting of all finite sequences or arrays of reals, naturals and booleans, together with certain standard constants and operations for the empty array, updating of arrays, etc.

The significance of arrays for computation is that they provide finite but unbounded memory. The reason for introducing the starred sort real* is the lack of effective coding of finite sequences from $\mathbb{R}$ (unlike the case of $\mathbb{N}$ ).

We make use of $\mathcal{R}^{*}$ to simplify one of our results. However, despite the convenience of the starred sort real*, the use of $\mathcal{R}^{*}$ is not essential as it is not strictly stronger for computation than $\mathcal{R}$ (we outline a proof of this fact in Appendix A). As such, we omit the precise definition of $\mathcal{R}^{*}$, which can be found in [TZ00, TZ15].

\subsection{The While programming language}

As has been mentioned, we will study the computation of functions and relations by high level imperative programming languages based on the ' while' construct.

For the remainder of this section, let $A$ be a standard algebra with signature $\Sigma$.

We begin with the syntax of $\Sigma$-terms.

Notation 2.7.1. We use 'Đ' to denote syntactic identity between two expressions.

Definition 2.7.2 ( $\Sigma$-variables). For each $\Sigma$-sort $s$, there are variables $\mathrm{x}^{s}, \mathrm{y}^{s}, \ldots$ of sort $s . \operatorname{Var}_{s}(\Sigma)$ is the set of variables of sort $s$, and $\operatorname{Var}(\Sigma)$ is the set of all $\Sigma$-variables. 
Definition 2.7.3 ( $\Sigma$-terms). $\boldsymbol{T} \boldsymbol{m}(\Sigma)$ is the set of $\Sigma$-terms: $t, \ldots$ and $\boldsymbol{T} \boldsymbol{m}_{s}(\Sigma)$ is the set of $\Sigma$-terms of sort $s: t^{s}, \ldots$. We define this using modified BNF:

$$
t^{s}::=\mathrm{x}^{s} \mid F\left(t_{1}^{s_{1}}, \ldots, t_{m}^{s_{m}}\right)
$$

where $F$ is a $\Sigma$-function symbol of type $s_{1} \times \ldots \times s_{m} \rightarrow s(m \geq 0)$.

Now we consider statements and procedures for the While language.

Definition 2.7.4 (Statements). $\boldsymbol{S t m t}(\Sigma)$ is the class of $\Sigma$-statements $S, \ldots$ generated by:

$$
S::=\text { skip }|\mathrm{x}:=t| S_{1} ; S_{2} \mid \text { if } b \text { then } S_{1} \text { else } S_{2} \text { fi } \mid \text { while } b \text { do } S_{0} \text { od }
$$

where $\mathrm{x}:=t$ denotes simultaneous assignment, i.e., for some $m>0$, $\mathrm{x} \equiv\left(\mathrm{x}_{1}, \ldots, \mathrm{x}_{m}\right)$ and $t \equiv\left(t_{1}, \ldots, t_{m}\right)$ are variable and term tuples of the same product type, with the condition that $\mathrm{x}_{i} \not \equiv \mathrm{x}_{j}$ for $i \neq j$; and $b$ is a boolean term.

Definition 2.7.5 (Procedures). $\operatorname{Proc}(\Sigma)$ is the class of $\Sigma$-procedures $P, \ldots$ of the form:

$$
P \equiv \operatorname{proc} D \text { begin } S \text { end }
$$

where the statement $S$ is the body and $D$ a variable declaration of the form

$$
D \equiv \text { in } \mathrm{a}: u \text { out } \mathrm{b}: v \text { aux } \mathrm{c}: w
$$

where $\mathrm{a}, \mathrm{b}$ and $\mathrm{c}$ are tuples of input variables, output variables and auxiliary variables respectively. We stipulate further:

(i) a, b and c each consist of distinct variables, and they are pairwise disjoint,

(ii) every variable occurring in the body $S$ must be declared in $D$ (among a, $\mathrm{b}$ or $\mathrm{c})$.

If $\mathrm{a}: u$ and $\mathrm{b}: v$, then $P$ has type $u \rightarrow v$, written $P: u \rightarrow v$.

For brevity, we will write $\boldsymbol{T} \boldsymbol{m}$ for $\boldsymbol{T m}(\Sigma)$, While for While $(\Sigma)$, etc. 


\section{$2.8 \quad$ States}

Definition 2.8.1 (State). A state is a family $\left\langle\sigma_{s} \mid s \in \boldsymbol{S o r t}(\Sigma)\right\rangle$ of functions $\sigma_{s}: \boldsymbol{V a r}_{s} \rightarrow A_{s}$. State $(A)$ is the set of states on $A$, with elements $\sigma, \ldots$.

Notation 2.8.2. We will write $\sigma(\mathrm{x})$ for $\sigma_{s}(\mathrm{x})$ when $\mathrm{x} \in \boldsymbol{V a r}_{s}$. We also write, for tuples $\mathrm{x} \equiv\left(\mathrm{x}_{1}, \ldots, \mathrm{x}_{m}\right), \sigma[\mathrm{x}]$ in place of $\left(\sigma\left(\mathrm{x}_{1}\right), \ldots, \sigma\left(\mathrm{x}_{m}\right)\right)$.

Definition 2.8.3 (Variant of a state). Let $\sigma$ be a state over $A$, and for some $\Sigma$-product type $u$, let $\mathrm{x} \equiv\left(\mathrm{x}_{1}, \ldots, \mathrm{x}_{n}\right)$ and $a=\left(a_{1}, \ldots, a_{n}\right) \in A^{u}($ for $n \geq 1)$. We define $\sigma\{\mathrm{x} / a\}$ to be the state over $A$ formed from $\sigma$ by replacing its value at $\mathrm{x}_{i}$ by $a_{i}$ for $i=1, \ldots, n$. That is, for all variables $\mathrm{y}$ :

$$
\sigma\{\mathrm{x} / a\}(\mathrm{y})= \begin{cases}\sigma(\mathrm{y}) & \text { if } \mathrm{y} \not \equiv \mathrm{x}_{i} \text { for } i=1, \ldots, n \\ a_{i} & \text { if } \mathrm{y} \equiv \mathrm{x}_{i}\end{cases}
$$

For $t \in \mathbf{T e r m}_{s}$, we will define the function

$$
\llbracket t \rrbracket^{A}: \operatorname{State}(A) \rightarrow A_{s}
$$

where $\llbracket t \rrbracket^{A} \sigma$ is the value of $t$ in $A$ at state $\sigma$.

Definition 2.8.4 (Semantics of terms). The definition of $\llbracket t \rrbracket^{A} \sigma$ is by structural induction on $\Sigma$-terms $t$ :

- $\llbracket \mathrm{x} \rrbracket^{A} \sigma=\sigma(\mathrm{x})$

- $\llbracket F\left(t_{1}, \ldots, t_{m}\right) \rrbracket^{A} \sigma \simeq \begin{cases}F\left(\llbracket t_{1} \rrbracket^{A} \sigma, \ldots, \llbracket t_{m} \rrbracket^{A} \sigma\right) & \text { if } \llbracket t_{i} \rrbracket^{A} \sigma \downarrow \text { for all } i=1, \ldots, m \\ \uparrow & \mathrm{o} / \mathrm{w}\end{cases}$

Notation 2.8.5. We write $\llbracket t \rrbracket^{A} \sigma \uparrow$ if it diverges and $\llbracket t \rrbracket^{A} \sigma \downarrow$ if it converges, which we can think of as the computation of $\llbracket t \rrbracket^{A} \sigma$ not halting and halting respectively. We also write $\llbracket t \rrbracket^{A} \sigma \downarrow a$ to mean that the evaluation of $\llbracket t \rrbracket^{A} \sigma$ converges to a value $a$. 
As with Kleene equality at the meta-level (Notation 2.5.2), we make use of Kleene equality on the meaning of terms.

Notation 2.8.6. $\llbracket t_{1} \rrbracket^{A} \sigma \simeq \llbracket t_{2} \rrbracket^{A} \sigma$ means that either $\llbracket t_{1} \rrbracket^{A} \sigma$ and $\llbracket t_{1} \rrbracket^{A} \sigma$ both converge to the same value, or both diverge (cf. [Kle52, §63]).

\subsection{Semantics of the While language}

In this section we define functions which give the meaning of While statements $S$ and procedures $P$, and some of the functions used to define those functions. The definitions of these functions is standard [TZ00, $\S 33.4-3.6, \S \S 3.14]$ and lengthy, and so we only give descriptions, not definitions.

We will make use of the computation step function:

$$
\operatorname{Comp}^{A}: \operatorname{Stmt} \times \operatorname{State}(A) \times \mathbb{N} \rightarrow \operatorname{State}(A) \cup\{*\} .
$$

$\boldsymbol{C o m p}^{A}(S, \sigma, n)$ is the $n$th step, or the state at the $n$th time cycle, in the computation of $S$ on $A$, starting at state $\sigma$. The symbol '*' indicates the computation is over.

From the computation step function we can easily define another function we will use, the computation length function:

$$
\operatorname{CompLen}^{A}: \operatorname{Stmt} \times \operatorname{State}(A) \rightarrow \mathbb{N}
$$

as follows:

$\operatorname{CompLen}^{A}(S, \sigma)=\left\{\begin{array}{ll}\text { least } n \text { such that } \boldsymbol{C o m p}^{A}(S, \sigma, n)=* & \text { if such an } n \text { exists } \\ \infty & \text { o/w }\end{array}\right.$.

We will also make use of the statement remainder function:

$$
\operatorname{Rem}^{A}: \text { Stmt } \times \text { State }(A) \times \mathbb{N} \rightarrow \text { Stmt } .
$$


$\boldsymbol{R e m}^{A}(S, \sigma, n)$ is the statement about to be executed at step $n$ of the computation of $S$ on $A$, starting at state $\sigma$ (or skip when the computation is over); this statement is called the "remainder" of $S$.

Now we may define the ideas of snapshots. We define the snapshot function:

$$
\operatorname{Snap}^{A}(S, \sigma, n): \boldsymbol{S t m t} \times \boldsymbol{S t a t e}(A) \times \mathbb{N} \rightarrow((\text { State }(A) \cup\{*\}) \times \text { Stmt })
$$

as

$$
\operatorname{Snap}^{A}(S, \sigma, n)=\left(\sigma^{n}, S^{n}\right)
$$

where

$$
\sigma^{n}=\boldsymbol{C o m p}^{A}(S, \sigma, n) \text { and } S^{n}=\boldsymbol{R e m}^{A}(S, \sigma, n) .
$$

$\operatorname{Snap}^{A}(S, \sigma, n)$ is the snapshot of the computation of $S$ at stage $n$, i.e. the pair $\left(\sigma^{n}, S^{n}\right)$ where $\sigma^{n}$ is the $n$th step in the computation of $S$ and $S^{n}$ is the statement about to be executed at state $n$.

From the snapshot function we may define the snapshot sequence generated by $S$ at $\sigma$ :

$$
(\boldsymbol{\sigma}, \boldsymbol{S})=\left(\left(\sigma^{0}, S^{0}\right),\left(\sigma^{1}, S^{1}\right),\left(\sigma^{2}, S^{2}\right), \ldots\right)
$$

$(\boldsymbol{\sigma}, \boldsymbol{S})$ is an infinite sequence of snapshots generated by $S$ at $\sigma$. If $S$ halts on $\sigma$, then eventually the sequence repeats $\left(\sigma^{\prime}\right.$, skip).

The meaning of a While statement $S$, written $\llbracket S \rrbracket^{A}$, is a partial state transformation on an algebra $A$ :

$$
\llbracket S \rrbracket^{A}: \text { State } \rightarrow \text { State } .
$$

Let $l=\operatorname{CompLen}^{A}(S, \sigma)$; then:

$$
\llbracket S \rrbracket^{A}(\sigma) \simeq\left\{\begin{array}{ll}
\boldsymbol{C o m p}^{A}(S, \sigma, l) & \text { if } l \neq \infty \\
\uparrow & \text { o/w }
\end{array} .\right.
$$


The meaning of a While procedure

$$
P \equiv \operatorname{proc} \text { in } \mathrm{a}: u \text { out } \mathrm{b}: v \text { aux c }: w \text { begin } S \text { end } u \rightarrow v
$$

is written $\llbracket P \rrbracket^{A}: A^{u} \rightarrow A^{v}$, and defined as follows. For $a \in A^{u}$, let $\sigma$ be any state on $A$ such that $\sigma[\mathrm{a}]=a$. Then

$$
\llbracket P \rrbracket^{A}(a) \simeq\left\{\begin{array}{ll}
\sigma^{\prime}[\mathrm{b}] & \text { if } \llbracket S \rrbracket^{A} \sigma \downarrow \sigma^{\prime} \text { (say) } \\
\uparrow & \text { if } \llbracket S \rrbracket^{A} \sigma \uparrow .
\end{array} .\right.
$$

\subsection{While computability and semicomputabil- ity}

Definition 2.10.1 (While computable function). Let $A$ be a standard algebra.

(a) A function $f: A^{u} \rightarrow A_{s}$ is said to be computable (on $A$ ) by a While procedure $P: u \rightarrow s$ if $f=P^{A}$.

(b) While $(A)$ is the class of functions While computable on $A$.

Definition 2.10.2 (Halting set). The halting set of a procedure $P: u \rightarrow v$ on $A$ is the set

$$
\boldsymbol{H a l t}^{A}(P)=_{d f}\left\{a \in A^{u} \mid P^{A}(a) \downarrow\right\}
$$

Definition 2.10.3 (While semicomputable set). A set $R \subseteq A^{u}$ is While semicomputable on $A$ if it is the halting set on $A$ for some While procedure.

\subsection{Extending While to While ${ }^{\mathrm{OR}}$ and While WN $^{\exists \mathrm{N}}$}

In preparation for the theorems in Chapter 4 and Chapter 5 , we give the semantics of strong disjunction and infinite disjunctions to introduce the extensions $\boldsymbol{W h i l e}^{\mathrm{OR}}$ and While $^{\exists \mathrm{N}}$ extensions of the While language. 
The motivation of these extensions is that our model of While computation on $\mathcal{R}$, the partial operations leave us unable to implement interleaving or dovetailing. The problem is that when interleaving two processes, one may converge and the other diverge locally (because of the partial operations). The resulting process will then diverge, whereas we would want it to converge. Thus, as we will see in one of the results of Chapter 4, the union of two semicomputable sets is not necessarily semicomputable! In concrete models do not have this deficiency. The extensions $\boldsymbol{W h} \boldsymbol{i l} \boldsymbol{e}^{\mathrm{OR}}$ and $\boldsymbol{W h i l e} \boldsymbol{E N}^{\exists \mathrm{N}}$ correct this deficiency.

The While WR $^{\mathrm{O}}$ language is created from While by introducing the strong (Kleene) disjunction operator ' $\nabla$ ', where $b_{1} \nabla b_{2}$ converges to true if either $b_{1}$ or $b_{2}$ converge to true, even if the other diverges.

The $\boldsymbol{W h i l e}^{\exists \mathrm{N}}$ language is created from While by introducing a strong existential quantification construct over the naturals:

$$
\mathrm{x}^{b}:=\exists \mathbf{z} P(t, \mathbf{z})
$$

where $z$ : nat and $P$ is a boolean valued procedure. Its semantics are as follows:

$$
\llbracket \exists \text { z } P(t, \mathrm{z}) \rrbracket^{A} \sigma \simeq \begin{cases}\text { true } & \text { if } P\left(\llbracket t \rrbracket^{A} \sigma, n\right) \downarrow \text { true for some } n \\ \uparrow & \mathrm{o} / \mathrm{w} .\end{cases}
$$

We also include the strong disjunction operator in the $\boldsymbol{W h i l e}^{\exists \mathrm{N}}$ language.

By means of these constructs, interleaving of processes may be simulated. The While $^{\mathrm{OR}}$ language allows for interleaving of an arbitrary but finite number of processes, and the While ${ }^{\exists \mathrm{N}}$ language allows for interleaving of infinitely many processes. 


\subsection{Extending While, While ${ }^{\mathrm{OR}}$ and While $^{\exists \mathrm{N}}$ to their starred versions}

Recall the algebra $\mathcal{R}^{*}$ (section 2.6). We now construct the While* $(\mathcal{R})$,

While $^{\mathrm{OR} *}(\mathcal{R})$ and While $^{\exists \mathrm{N} *}(\mathcal{R})$.

Definition 2.12.1 (Simple and starred variables). We call the variables of sort real, nat and bool simple, and the variables of sort real*, nat* and bool* starred.

Definition 2.12.2 (The While* $(\mathcal{R})$ language). A While $^{*}(\mathcal{R})$, While OR* $^{\mathrm{R}}(\mathcal{R})$ or While $^{\exists \mathrm{N} *}(\mathcal{R})$ procedure is respectively a $\boldsymbol{W h i l e}\left(\mathcal{R}^{*}\right)$, While $\mathrm{OR}\left(\mathcal{R}^{*}\right)$ or While $^{\exists \mathrm{N}}\left(\mathcal{R}^{*}\right)$ procedure for which the input and output variables are simple. However, the auxiliary variables may be starred.

\subsection{Encoding of syntax}

We assume given a family of effective numerical codings for each of the classes of syntactic expressions over $\Sigma$. We write $\ulcorner E\urcorner$ for the code of an expression $E$. We make the following assumptions about the coding:

- $\ulcorner E\urcorner$ increases strictly with the complexity of $E$, and so (e.g.), the code of an expression is larger than those of its subexpressions.

- Sets of codes of the various syntactic classes, and of their respective subclasses, such as $\{\ulcorner t\urcorner \mid t \in \boldsymbol{T e r m}\},\left\{\ulcorner t\urcorner \mid t \in \boldsymbol{T e r m}_{s}\right\},\{\ulcorner S\urcorner \mid S \in \boldsymbol{S t m t}\}$, $\{\ulcorner S\urcorner \mid S$ is an assignment $\}$, etc., are primitive recursive.

- We can go primitive recursively from codes of expressions to codes of their immediate subexpressions and vice versa; thus, for example, $\left\ulcorner S_{1}\right\urcorner$ and $\left\ulcorner S_{2}\right\urcorner$ are primitive recursive in $\left\ulcorner S_{1} ; S_{2}\right\urcorner$, and conversely. 
In short, we can primitive recursively simulate all operations involved in processing the syntax of the programming language. 


\section{Chapter 3}

\section{Semantic disjointedness;}

\section{Engeler's Lemma}

\subsection{Engeler's Lemma}

The following lemma is of vital importance to proving our Structure Theorem for $\operatorname{While}(\mathcal{R})$ semicomputable sets [Eng68], [TZ00].

Lemma 3.1.1 (Engeler's Lemma for While). If a relation $R \subseteq A^{u}$ is While semicomputable over a standard partial $\Sigma$-algebra $A$, then $R$ can be expressed as the disjunction of an effective countable sequence of $\Sigma$-booleans ${ }^{1}$ over $A$.

i.e.,

$$
x \in R \Longleftrightarrow \bigvee_{k=0}^{\infty} b_{k}[x]
$$

for an effective sequence for booleans $\left(b_{1}, b_{2}, b_{3}, \ldots\right)$.

We also need the following concept and lemma.

${ }^{1}$ That is, $\Sigma$-terms of sort bool. 
Definition 3.1.2 (Semantic Disjointedness). A sequence $\left(b_{0}, b_{1}, b_{2}, \ldots\right)$ of boolean terms is semantically disjoint over $A$ if for any state $\sigma$ over $A$ and any $n$,

$$
\llbracket b_{n} \rrbracket^{A} \sigma \downarrow \text { true } \Longrightarrow \forall i \neq n, \llbracket b_{i} \rrbracket^{A} \sigma \downarrow \text { false. }
$$

The following lemma was proved in [XFZ15, §4].

Lemma 3.1.3 (Semantic Disjointedness Lemma). The sequence of computable boolean terms generated from a While computation tree $S$ by the construction using computation trees in the proof of Engeler's Lemma ${ }^{2}$ is semantically disjoint.

\subsection{Canonical form for booleans over $\mathcal{R}$}

In our proof of our Structure Theorem for $\operatorname{While}(\mathcal{R})$ semicomputable sets, we require a canonical form for booleans over $\mathcal{R}$.

Lemma 3.2.1 (Canonical form for booleans over $\mathcal{R}$ ). An $\mathcal{R}$-boolean with variables of sort real only is effectively semantically equivalent to a boolean combination of equations and inequalities of the form:

$$
p(x)=0 \text { and } q(x)>0
$$

where $p$ and $q$ are polynomials in $x$ of degree $>0$.

The proof of Lemma 3.2.1 resembles that of a similar lemma for booleans over $\mathcal{R}^{\mathrm{OR}}[\mathrm{Fu} 14, \S \S 4.1]$.

\subsection{Semi-algebraic and basic sets}

We introduce the concepts of semi-algebraic and basic sets, which are fundamental to our results. We consider these sets on $\mathbb{R}^{2}$, though they can clearly be generalised to $\mathbb{R}^{n}$ for any $n \geq 0$.

\footnotetext{
${ }^{2}[$ XFZ15, Lemma 4.3.1]
} 
Definition 3.3.1 (Semi-algebraic set). A semi-algebraic set is a finite union of sets of the form

$$
\left\{x \in \mathbb{R}^{2} \mid p_{1}(x)>0, \ldots, p_{k}(x)>0, q_{1}(x)=0, \ldots, q_{l}(x)=0\right\}(k, l \geq 0)
$$

where $p_{1}, \ldots, p_{k}, q_{1}, \ldots, q_{l}$ are polynomials with integer coefficients.

Definition 3.3.2 (Basic set). A basic set ${ }^{3}$ is a particular kind of semi-algebraic set, of the form

$$
\left\{x \in \mathbb{R}^{2} \mid p_{1}(x)>0, \ldots, p_{k}(x)>0\right\}(k \geq 0)
$$

where $p_{1}, \ldots, p_{k}$ are polynomials with integer coefficients.

Remark 3.3.3. All basic sets are open.

Remark 3.3.4. Basic (open) sets are closed under intersection.

Lemma 3.3.5. Given a polynomial $p(\mathrm{x})$ on $\mathbb{R}^{2}$, there are disjoint basic sets $B^{+}, B^{-}$and a semi-algebraic set $D$, such that on $B^{+}, p>0$, on $B^{-}, p<0$, and on $D, p=0$, and $B^{+} \cup B^{-} \cup D=\mathbb{R}^{2}$

Proof. Clear.

\subsection{Positive and negative sets}

Notation 3.4.1. For a pair of variables $\mathrm{x} \equiv\left(\mathrm{x}_{1}, \mathrm{x}_{2}\right)$ : real ${ }^{2}$, let $\boldsymbol{B} \boldsymbol{o o l}(\mathrm{x})$ be the set of $\Sigma(\mathcal{R})$-booleans with no free variables other than $\mathrm{x}$.

As mentioned in Remark 2.5.4, we focus entirely on functions over $\mathbb{R}^{2}$ throughout this section.

We use the notions of positive, negative and divergent sets of booleans:

\footnotetext{
${ }^{3}$ In some texts, basic sets as we define them are called basic open sets; we work only with basic open sets, and so often omit the "open".
} 
Definition 3.4.2. For any $b \in \boldsymbol{B} \boldsymbol{B o o l}(\mathrm{x})$, let:

$$
\begin{gathered}
P S(b)=_{d f}\left\{x \in \mathbb{R}^{2} \mid b[x]=\text { true }\right\} \\
N S(b)=_{d f}\left\{x \in \mathbb{R}^{2} \mid b[x]=\text { false }\right\} \\
D S(b)=_{d f}\left\{x \in \mathbb{R}^{2} \mid b[x] \uparrow\right\} .
\end{gathered}
$$

We call $P S(b), N S(b)$ and $D S(b)$ the positive, negative and divergent sets of $b$ respectively. 


\section{Chapter 4}

\section{While $(\mathcal{R})$ semicomputable}

\section{sets: Structure Theorem}

\section{and failure of closure under}

\section{union}

In this chapter we begin by extending the Partition Lemma for While $(\mathcal{R})$ semi-

computability given in [Fu14], which we then use to give a Structure Theorem for While $(\mathcal{R})$ semicomputability. Then, using that Structure Theorem, we give an example of two $\operatorname{While}(\mathcal{R})$ semicomputable sets whose union is not $\boldsymbol{W h i l e}(\mathcal{R})$, semicomputable, thus disproving the closure of $\operatorname{While}(\mathcal{R})$ semicomputable sets under union. 


\subsection{Partition Lemma for While $(\mathcal{R})$}

In addition to the lemmas given in chapter 3 , we require one additional lemma in order to prove our Structure Theorem for While $(\mathcal{R})$ semicomputable sets. This Partition Lemma is a strengthening of a similar lemma given in [Fu14, $\S \S 4.3]$, made possible by restricting our attention to While.

Lemma 4.1.1 (Partition Lemma for booleans on $\mathcal{R}$ ). Consider any boolean $b \in \boldsymbol{B o o l}(\mathrm{x}) .{ }^{1}$ We may construct positive and negative sets ${ }^{2}$ for $b$ expressed as:

$$
\begin{gathered}
P S(b)=\bigcup_{i=1}^{k} B_{i}^{+} \\
N S(b)=\bigcup_{j=1}^{l} B_{j}^{-} .
\end{gathered}
$$

where $B_{i}^{+}, B_{j}^{-}$are basic (open) sets, and

$$
\begin{aligned}
& B_{i}^{+} \cap B_{j}^{-}=\emptyset \text { for } i=1, \ldots, k \text { and } j=1, \ldots, l \\
& B_{i_{1}}^{+} \cap B_{i_{2}}^{+}=\emptyset \text { for } i_{1} \neq i_{2} \\
& B_{j_{1}}^{-} \cap B_{j_{2}}^{-}=\emptyset \text { for } j_{1} \neq j_{2}
\end{aligned}
$$

Remark 4.1.2. In [XFZ15] and [Fu14], basic sets for $b$ were constructed such that only the first of the intersection properties hold, but for our purposes, considering the specific case of the While language, it is important to explicitely have all three.

\footnotetext{
${ }^{1}$ Notation 3.4.1

${ }^{2}$ For our purpose, the form of the divergent set of $b, D S(b)$, is unimportant.
} 
Discussion 4.1.3. Before giving the proof, we first consider some examples of positive and negative sets in $\mathbb{R}^{2}$.

Consider the polynomials $p_{1}=-x_{1}^{2}-x_{2}^{2}+1$ and $p_{2}=-\left(x_{1}-1\right)^{2}-x_{2}^{2}+1$, and the booleans $b_{1} \equiv p_{1}\left(x_{1}, x_{2}\right)>0$ and $b_{2} \equiv p_{2}\left(x_{1}, x_{2}\right)>0$. Then:

$$
\begin{aligned}
& B_{1}=P S\left(b_{1}\right)=\left\{\left(x_{1}, x_{2}\right) \mid p_{1}\left(x_{1}, x_{2}\right)>0\right\} \\
& B_{2}=P S\left(b_{2}\right)=\left\{\left(x_{1}, x_{2}\right) \mid p_{2}\left(x_{1}, x_{2}\right)>0\right\}
\end{aligned}
$$

Figure 4.1: $B_{1}=P S\left(b_{1}\right)$

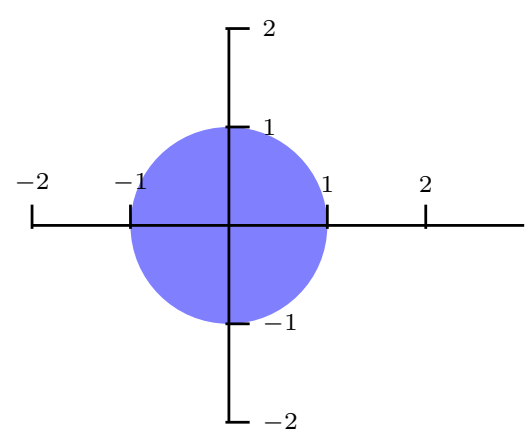

Figure 4.2: $B_{2}=P S\left(b_{2}\right)$

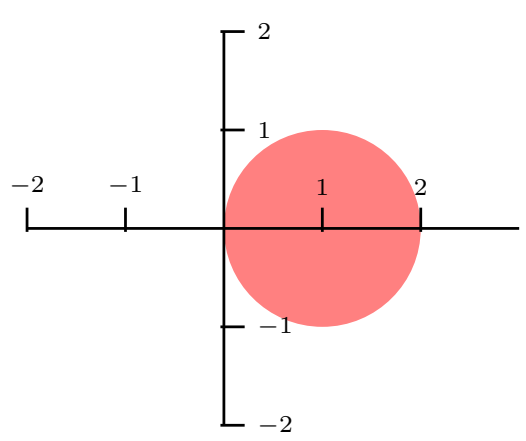

$B_{1}$ and $B_{2}$ are basic sets, and can be easily be seen to be $\boldsymbol{W h i l e}(\mathcal{R})$ semicomputable. They are pictured in Figures 4.1 and 4.2 respectively. 
Figure 4.3: $P S\left(b_{1} \stackrel{c}{\vee} b_{2}\right)$

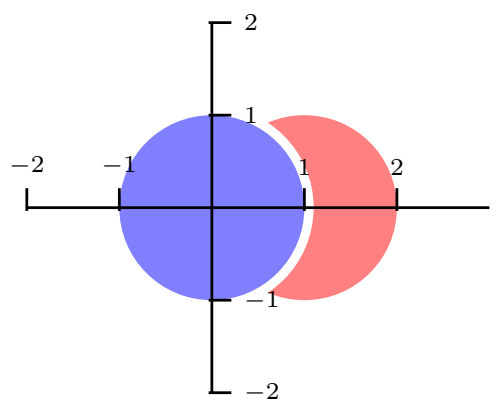

Figure 4.4: $P S\left(b_{1} \vee b_{2}\right)$

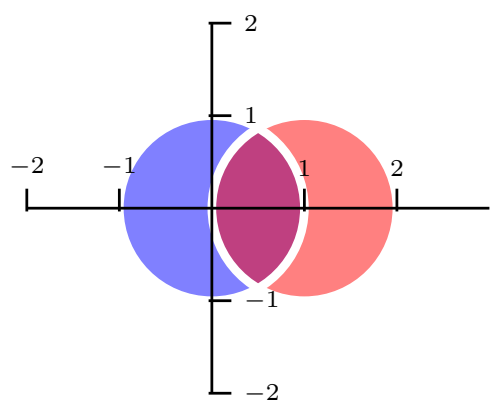

The sets $P S\left(b_{1} \stackrel{c}{\vee} b_{2}\right)$ and $P S\left(b_{1} \vee b_{2}\right)$, pictured in Figures 4.3 and 4.4 respectively, are easily seen to be semicomputable. These sets can be represented as the union of two and three disjoint basic sets respectively ${ }^{3}$ :

$$
\begin{aligned}
P S\left(b_{1} \vee b_{2}\right)= & \left\{\left(x_{1}, x_{2}\right) \mid p_{1}\left(x_{1}, x_{2}\right)>0\right\} \\
& \cup\left\{\left(x_{1}, x_{2}\right) \mid p_{2}\left(x_{1}, x_{2}\right)>0, p_{1}\left(x_{1}, x_{2}\right)<0\right\} \\
P S\left(b_{1} \vee b_{2}\right)= & \left\{\left(x_{1}, x_{2}\right) \mid p_{1}\left(x_{1}, x_{2}\right)>0, p_{2}\left(x_{1}, x_{2}\right)<0\right\} \\
& \cup\left\{\left(x_{1}, x_{2}\right) \mid p_{2}\left(x_{1}, x_{2}\right)>0, p_{1}\left(x_{1}, x_{2}\right)<0\right\} \\
& \cup\left\{\left(x_{1}, x_{2}\right) \mid p_{2}\left(x_{1}, x_{2}\right)>0, p_{1}\left(x_{1}, x_{2}\right)>0\right\}
\end{aligned}
$$

\footnotetext{
${ }^{3}$ The proof of Lemma 4.1.1 serves as an algorithm for constructing such representations.
} 
We proceed to the proof of the Partition Lemma.

Proof of the Partition Lemma for booleans on $\mathcal{R}$ (Lemma 4.1.1). By structural induction on the canonical form of booleans on $\mathcal{R}$.

Base case: $b \equiv p(\mathrm{x})=0$ or $p(\mathrm{x})>0$. Immediate from Lemma 3.3.5, because in each case there is a single basic set for each of the positive and negative sets respectively.

Induction step:

In what follows, suppose:

$$
\begin{aligned}
& P S\left(b_{1}\right)=\bigcup_{i=1}^{k_{1}} B_{1 i}^{+} \\
& N S\left(b_{1}\right)=\bigcup_{i=1}^{l_{1}} B_{1 i}^{-} \\
& P S\left(b_{2}\right)=\bigcup_{j=1}^{k_{2}} B_{2 j}^{+} \\
& N S\left(b_{2}\right)=\bigcup_{j=1}^{l_{2}} B_{2 j}^{-}
\end{aligned}
$$

Now we consider the various cases based on the canonical form of $b$ :

(i) $b \equiv \neg b_{1}$. Then just exchange the positive and negative sets of $b_{1}$; since all three properties hold for both, they still hold after switching.

(ii) $b \equiv b_{1} \vee b_{2}$. Then

$$
\begin{aligned}
& P S(b)=\left(\bigcup_{i=1}^{k_{1}} \bigcup_{j=1}^{k_{2}}\left(B_{1 i}^{+} \cap B_{2 j}^{+}\right)\right) \cup\left(\bigcup_{i=1}^{k_{1}} \bigcup_{j=1}^{l_{2}}\left(B_{1 i}^{+} \cap B_{2 j}^{-}\right)\right) \cup\left(\bigcup_{i=1}^{l_{1}} \bigcup_{j=1}^{k_{2}}\left(B_{1 i}^{-} \cap B_{2 j}^{+}\right)\right) \\
& N S(b)=\bigcup_{i=1}^{l_{1}} \bigcup_{j=1}^{l_{2}}\left(B_{1 i}^{-} \cap B_{2 j}^{-}\right)
\end{aligned}
$$

The outer union of $P S(b)$ is disjoint, because $B_{i}^{+} \cap B_{j}^{-}=\emptyset$. Further, the inner unions of $P S(b)$ and the union of $N S(b)$ are disjoint, because $B_{i_{1}}^{+} \cap B_{i_{2}}^{+}=\emptyset$ for $i_{1} \neq i_{2}$, and $B_{j_{1}}^{-} \cap B_{j_{2}}^{-}=\emptyset$ for $j_{1} \neq j_{2}$.

So $P S(b)$ and $N S(b)$ are finite unions of disjoint sets, all of which are basic, as the intersection of any two basic sets is also basic (Remark 3.3.4). 
(iii) $b \equiv b_{1} \wedge b_{2}$. Then

$$
\begin{aligned}
& P S(b)=\bigcup_{i=1}^{k_{1}} \bigcup_{j=1}^{k_{2}}\left(B_{1 i}^{+} \cap B_{2 j}^{+}\right) \\
& N S(b)=\left(\bigcup_{i=1}^{l_{1}} \bigcup_{j=1}^{l_{2}}\left(B_{1 i}^{-} \cap B_{2 j}^{-}\right)\right) \cup\left(\bigcup_{i=1}^{k_{1}} \bigcup_{j=1}^{l_{2}}\left(B_{1 i}^{+} \cap B_{2 j}^{-}\right)\right) \cup\left(\bigcup_{i=1}^{l_{1}} \bigcup_{j=1}^{k_{2}}\left(B_{1 i}^{-} \cap B_{2 j}^{+}\right)\right)
\end{aligned}
$$

Similar to case (ii), we observe that $P S(b)$ and $N S(b)$ are unions of disjoint basic sets.

(iv) $b \equiv b_{1} \stackrel{c}{\vee} b_{2}$. Then

$$
\begin{aligned}
& P S(b)=\bigcup_{i=1}^{k_{1}} B_{1 i}^{+} \cup\left(\bigcup_{i=1}^{l_{1}} \bigcup_{j=1}^{k_{2}}\left(B_{1 i}^{-} \cap B_{2 j}^{+}\right)\right) \\
& N S(b)=\bigcup_{i=1}^{l_{1}} \bigcup_{j=1}^{l_{2}}\left(B_{1 i}^{-} \cap B_{2 j}^{-}\right)
\end{aligned}
$$

Again similar to case (ii).

(v) $b \equiv b_{1} \stackrel{c}{\wedge} b_{2}$. Then

$$
\begin{aligned}
& P S(b)=\bigcup_{i=1}^{k_{1}} \bigcup_{j=1}^{k_{2}}\left(B_{1 i}^{+} \cap B_{2 j}^{+}\right) \\
& N S(b)=\bigcup_{i=1}^{k_{1}} B_{1 i}^{-} \cup\left(\bigcup_{i=1}^{k_{1}} \bigcup_{j=1}^{l_{2}}\left(B_{1 i}^{+} \cap B_{2 j}^{-}\right)\right)
\end{aligned}
$$

Again similar to (ii).

Remark 4.1.4. The Partition Lemma for booleans on $\mathcal{R}$ (Lemma 4.1.1) does

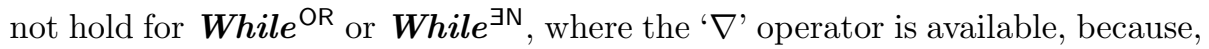
given any two booleans $b_{1}, b_{2} \in \boldsymbol{B} \boldsymbol{\operatorname { o o l }}(\mathrm{x})$, we cannot necessarily reduce the positive set of $b_{1} \nabla b_{2}$ to a disjoint union, as we will see in section 4.3. 


\subsection{Structure Theorem for While $(\mathcal{R})$ semicom- putability}

From $[$ Fu14, $§ 4.6]$, we have the following lemma ${ }^{4}$ for $\boldsymbol{W h i l e}(\mathcal{R})$ semicomputability:

Lemma 4.2.1. For subsets of $\mathbb{R}^{2}$,

(a) While $(\mathcal{R})$ s/comp $\Longrightarrow$ countable union of effective disjoint sequence

of finite unions of basic sets

(b) countable union of effective disjoint sequence $\Longrightarrow$ While $(\mathcal{R})$ s/comp.

of basic sets

We now strengthen this lemma by restricting the sequence in part (a) to basic sets rather than finite unions of basic sets, which provides us with an equivalence:

Theorem 1 (Structure Theorem for While $(\mathcal{R})$ ). For subsets of $\mathbb{R}^{2}$,

While $(\mathcal{R}) s /$ comp $\Longleftrightarrow$ countable union of an effective disjoint sequence of basic sets.

Proof. The ' $\Longleftarrow$ ' direction is simply part (b) of Lemma 4.2.1

For the ' $\Longrightarrow$ ' direction, we strengthen part (a) of Lemma 4.2.1 as follows:

If $R \subseteq \mathbb{R}^{2}$ is While $(\mathcal{R})$ semicomputable, then by Engeler's Lemma (Lemma 3.1.1), for all $x \in \mathbb{R}^{2}$,

$$
x \in R \Longleftrightarrow \bigvee_{k=0}^{\infty} b_{k}[x]
$$

for an effective sequence $\left(b_{k}\right)$ of $\Sigma$-booleans in $\boldsymbol{B o o l}(\mathrm{x})$. By the Partition Lemma, each $b_{k}$ defines a finite union of effective disjoint basic sets.

\footnotetext{
${ }^{4}$ Lemma 4.2.1 was presented as a Structure Theorem for While $(\mathcal{R})$ in [Fu14].
} 
By the Semantic Disjointedness Lemma (Lemma 3.1.3), the sequence $\left(b_{k}\right)$ is semantically disjoint over $\mathcal{R}$, and hence the positive sets for different $b_{k}$ 's are disjoint.

Hence $b_{k}$ is a disjoint sequence of effective basic sets as desired.

\subsection{Failure of closure of While $(\mathcal{R})$ semicomputable sets under union}

For total standard algebras, we have the following lemma [TZ00, §§5.2], [TZ15, $\S \S 6.1]:$

Lemma 4.3.1 (Closure of While semicomputable sets under union for total standard algebras). For any total standard algebra $A$, the class of While $(A)$ semicomputable sets is closed under finite unions.

We may use the Structure Theorem for $\boldsymbol{W h i l e}(\mathcal{R})($ Theorem 1) to easily give a counterexample to the closure of semicomputable sets under finite union the partial algebra of the reals.

Theorem 2 (Failure of closure of While $(\mathcal{R})$ semicomputable sets under union).

The class of While $(\mathcal{R})$ semicomputable sets on $\mathbb{R}^{2}$ is not closed under finite unions.

For the proof, we require the following discussion:

Discussion 4.3.2 (A union of two basic sets which is not basic). Consider the overlapping basic sets:

$$
\begin{aligned}
& B_{1}=\left\{\left(x_{1}, x_{2}\right) \mid-x_{1}^{2}-x_{2}^{2}+1>0\right\}, \\
& B_{2}=\left\{\left(x_{1}, x_{2}\right) \mid-\left(x_{1}-1\right)^{2}-x_{2}^{2}+1>0\right\}
\end{aligned}
$$

discussed in Discussion 4.1.3. 
Their union is clearly semi-algebraic, but not basic.

This follows from a more general result from [ABR96]:

If the boundaries of two semi-algebraic subsets of $\mathbb{R}^{2}$ intersect transversally at some point, then their union is never basic ${ }^{5}$.

\section{Proof of Theorem 2.}

Figure 4.5: $B_{1} \cup B_{2}=P S\left(b_{1} \nabla b_{2}\right)$

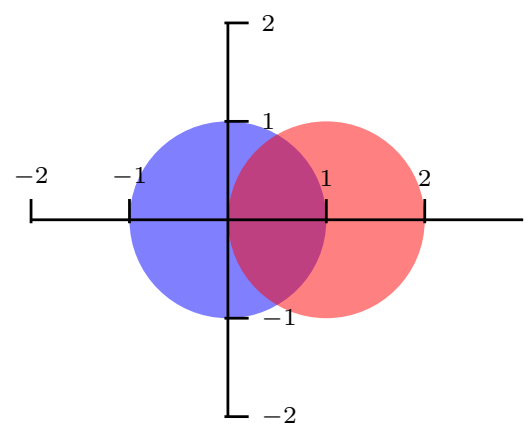

Recall again the sets $B_{1}$ and $B_{2}$ from Discussion 4.1.3. Consider $B_{1} \cup B_{2}=P S\left(b_{1} \nabla b_{2}\right)$, pictured in Figure 4.5. It is a union of two semicomputable sets (pictured in Figures 4.1 and 4.2). If it is semicomputable, then by the Structure Theorem for While it is an effective disjoint sequence of basic sets. However, since it is open and connected, it must in fact be equal to a single basic set. However, by Discussion 4.3.2, there is no way to represent $B_{1} \cup B_{2}$ as a single basic sets. So while $B_{1}$ and $B_{2}$ are semicomputable, their union is not.

Note that with respect to While $^{\mathrm{OR}}(\mathcal{R})$ and While $^{\exists \mathrm{N}}(\mathcal{R})$, the set $P S\left(b_{1} \nabla b_{2}\right)$ is trivially semicomputable (cf. Remark 4.1.4).

\footnotetext{
${ }^{5}$ We thank Professor Bröcker (Münster) for clarifying this (personal communication).
} 
Discussion 4.3.3. While it is intuitively clear that $B_{1} \cup B_{2}$ is not basic, this fact still requires a proof, as outlined in Discussion 4.3.2. To underline this, we will now consider a brief example of a set which seems intuitively not basic, but in fact is.

Consider the basic sets:

$$
\begin{aligned}
& B_{3}=P S\left(\left\{\left(x_{1}, x_{2}\right) \mid-2<x_{1}<-1 \wedge 1<x_{2}<2\right\}\right) \\
& B_{4}=P S\left(\left\{\left(x_{1}, x_{2}\right) \mid 1<x_{1}<2 \wedge 1<x_{2}<2\right\}\right) \\
& B_{5}=P S\left(\left\{\left(x_{1}, x_{2}\right) \mid-2<x_{1}<-1 \wedge-2<x_{2}<-1\right\}\right) \\
& B_{6}=P S\left(\left\{\left(x_{1}, x_{2}\right) \mid 1<x_{1}<2 \wedge-2<x_{2}<-1\right\}\right)
\end{aligned}
$$

Figure 4.6: $B_{3} \cup B_{4} \cup B_{5} \cup B_{6}$

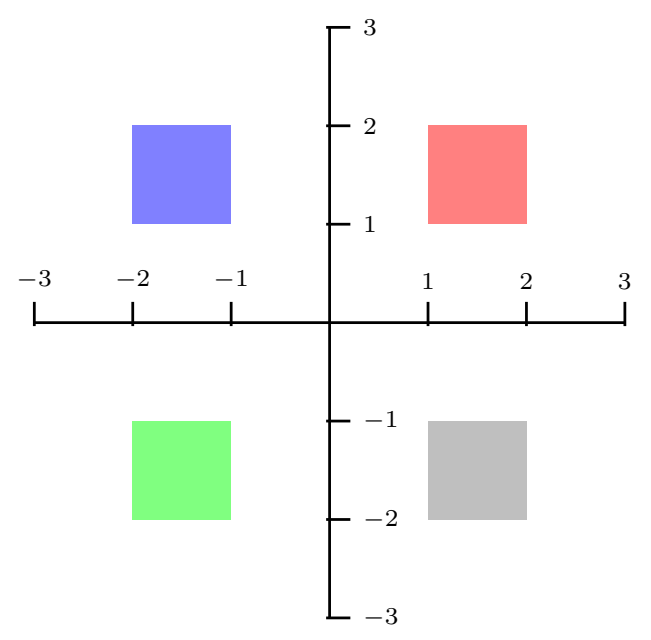


Their union, pictured in Figure 4.6, seems intuitively not basic (though clearly it is semi-algebraic), if it is written in the obvious way:

$$
\begin{aligned}
B_{3} \cup B_{4} \cup B_{5} \cup B_{6}=P S\left(\left\{\left(x_{1}, x_{2}\right)\right.\right. & \mid\left(-2<x_{1}<-1 \wedge 1<x_{2}<2\right) \\
& \vee\left(1<x_{1}<2 \wedge 1<x_{2}<2\right) \\
& \vee\left(-2<x_{1}<-1 \wedge-2<x_{2}<-1\right) \\
& \left.\left.\vee\left(1<x_{1}<2 \wedge-2<x_{2}<-1\right)\right\}\right)
\end{aligned}
$$

However, the union is, in fact, basic, since it can be written as:

$$
B_{3} \cup B_{4} \cup B_{5} \cup B_{6}=P S\left(\left\{\left(x_{1}, x_{2}\right) \mid 1<x_{1}^{2}<4 \wedge 1<x_{2}^{2}<4\right\}\right)
$$




\section{Chapter 5}

\section{Classes of sets}

\section{semicomputable by models}

\section{based on the While}

\section{language}

In this chapter we consider the equivalence or inequivalence of the classes of While $(\mathcal{R})$, While $\left.\mathrm{OR}^{\mathrm{R}}\right)$ and $\boldsymbol{W h i l e}^{\exists \mathrm{N}}(\mathcal{R})$ semicomputable sets, as well as the semicomputable sets of the projective versions of those languages.

5.1 A set which is projectively $\operatorname{While}(\mathcal{R})$ semicomputable but not While $(\mathcal{R})$ semicomputable

We will show that projective $\boldsymbol{W h i l e}(\mathcal{R})$ semicomputability is strictly stronger than While $(\mathcal{R})$ semicomputability by giving a set which is projectively $\boldsymbol{W h i l e}(\mathcal{R})$ 
semicomputable but not While $(\mathcal{R})$ semicomputable.

Figure 5.1: Domain of $f_{0}\left(x_{1}, x_{2}, y\right)$.

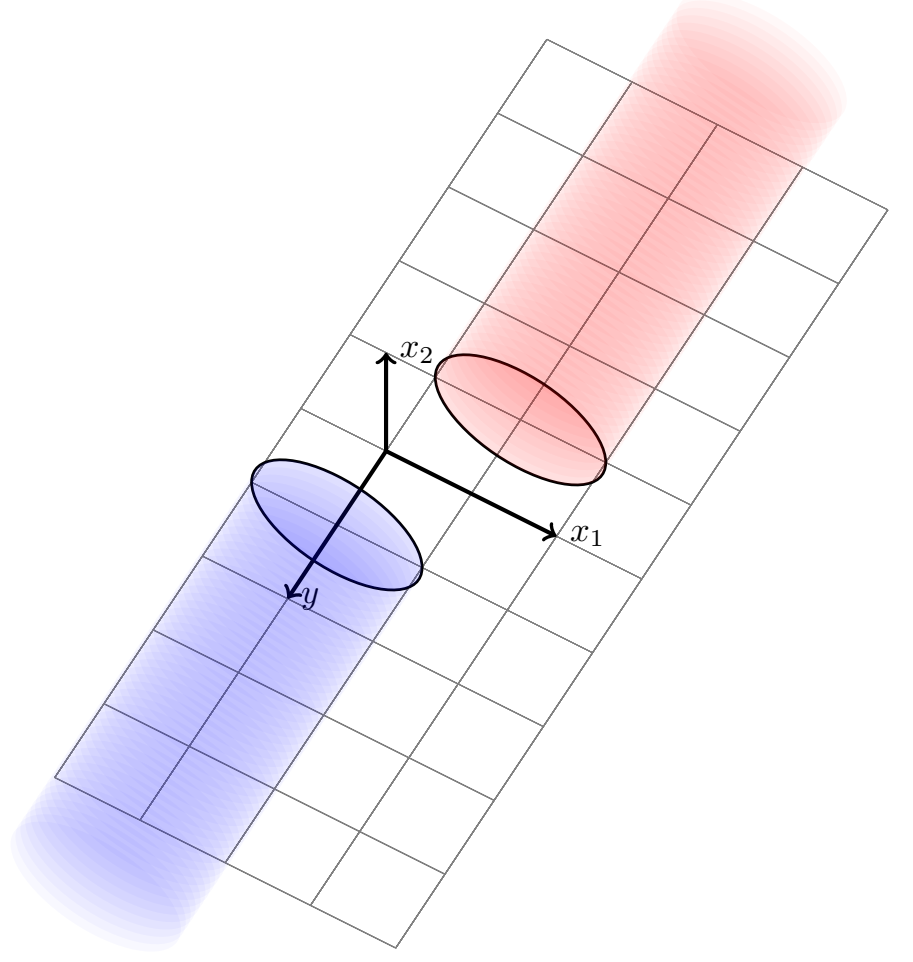

Consider the three-dimensional boolean valued function:

$$
f_{0}\left(x_{1}, x_{2}, y\right)= \begin{cases}x_{1}^{2}+x_{2}^{2}<1 & \text { if } y>1 \\ \left(x_{1}-1\right)^{2}+x_{2}^{2}<1 & \text { if } y<-1 \\ \uparrow & \text { o/w }\end{cases}
$$

the domain of which is pictured in Figure 5.1.

The domain of $f_{0}$ is easily seen to be $\boldsymbol{W h i l e}(\mathcal{R})$ semicomputable, and so its projection off the third argument:

$$
f\left(x_{1}, x_{2}\right)=_{d f} \exists y: \mathbb{R},\left(y<-1 \wedge x_{1}^{2}+x_{2}^{2}<1\right) \vee\left(y>1 \wedge\left(x_{1}-1\right)^{2}+x_{2}^{2}<1\right)
$$

is projectively $\boldsymbol{W h i l e}(\mathcal{R})$ semicomputable. 
We have seen this set previously in Figure 4.5, and we have seen that it is not While $(\mathcal{R})$ semicomputable (during the proof of Theorem 2), as it is not a union of disjoint basic sets.

From this example, we have the following theorem:

Theorem 3. For subsets of $\mathbb{R}^{2}$,

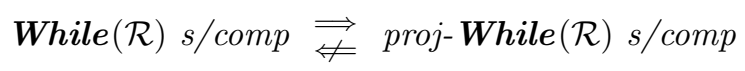

Proof. The ' $\Longrightarrow$ ' direction is clear from the definition of projective $\boldsymbol{W h i l e}(\mathcal{R})$ semicomputability. The ' $\Longleftarrow$ ' direction is clear from the above.

Note that this set is $\boldsymbol{W h i l e}^{\mathrm{OR}}(\mathcal{R})$ semicomputable, from the following theorem given in $[\mathrm{Fu} 14, \S \S 4.6]$ :

Theorem (Structure Theorem for While ${ }^{\mathrm{OR}}(\mathcal{R})$ ). For subsets of $\mathbb{R}^{2}$,

While $^{\mathrm{OR}}(\mathcal{R}) s /$ comp $\Longleftrightarrow$ countable union of an effective disjoint sequence of finite unions of basic sets.

Hence we can also compare $\boldsymbol{W h i l e}(\mathcal{R})$ and $\boldsymbol{W h i l e}^{\mathrm{OR}}(\mathcal{R})$ semicomputable sets:

Theorem 4. For subsets of $\mathbb{R}^{2}$,

$$
\text { While }(\mathcal{R}) s / \text { comp } \underset{\text { While }}{\mathrm{OR}}(\mathcal{R}) \text { s/comp }
$$




\subsection{A set which is projectively While $^{\mathrm{OR}}(\mathcal{R})$ semi- computable but not While $^{\mathrm{OR}}$ semicomputable}

We will now show a similar example which proves that projective While $^{\mathrm{OR}}(\mathcal{R})$ semicomputability is stronger that While $^{\mathrm{OR}}(\mathcal{R})$ semicomputability.

Figure 5.2: Domain of $g_{0}\left(x_{1}, x_{2}, n\right)$.
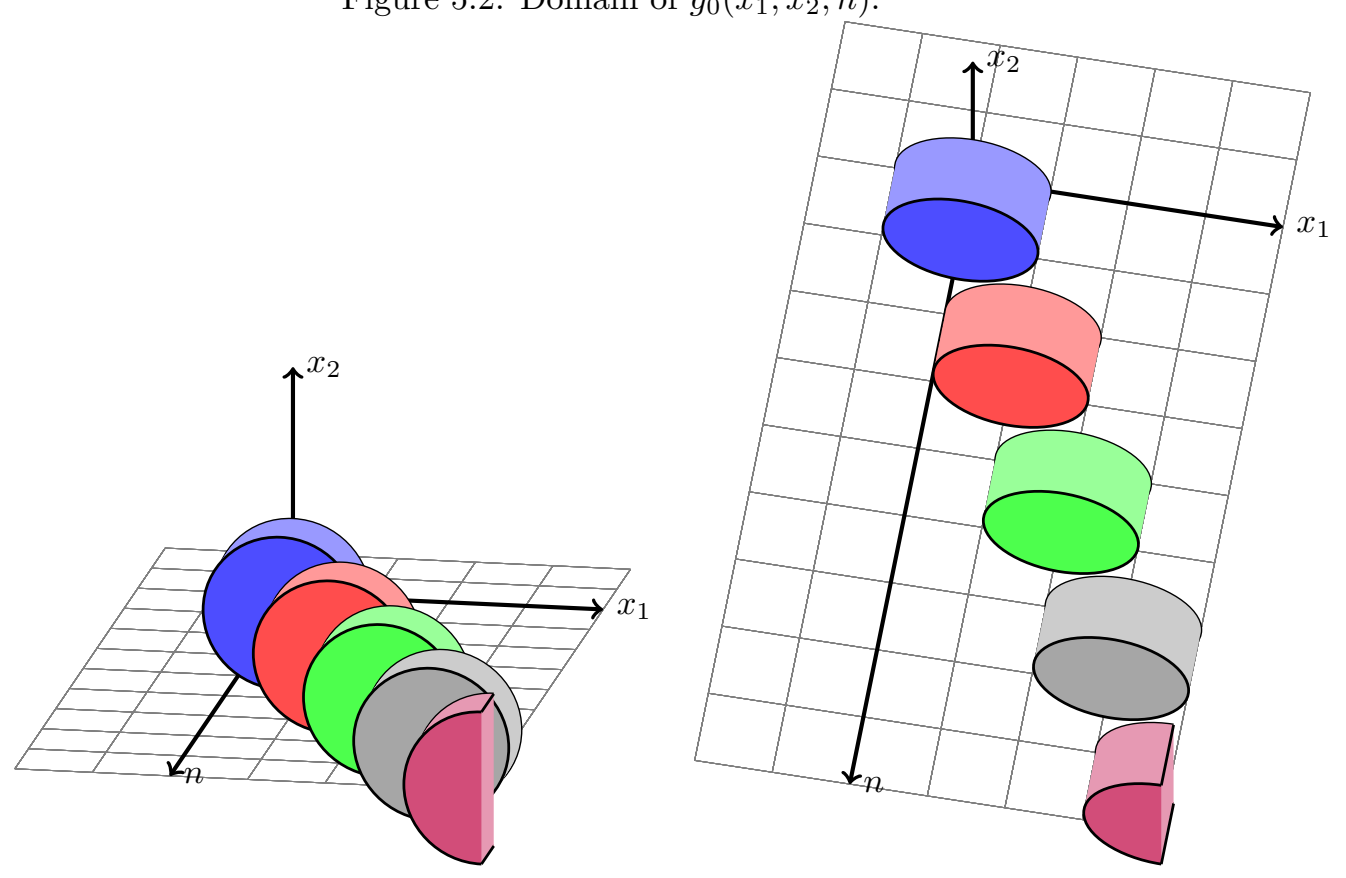

Consider the three dimensional boolean valued function:

$$
g_{0}\left(x_{1}, x_{2}, n\right)=\left(x_{1}-2 n\right)^{2}+x_{2}^{2}<1
$$

the domain of which is partially pictured from two viewpoints in Figure 5.2, for $x_{1} \leq 4$. This function describes an infinite sequence of disjoint discs of depth 1 whose projections overlap in the $x_{1}-x_{2}$ plane. 
Now, much as with the example of $f_{0}$ in section 5.1 , we can easily observe the the domain of $g_{0}$ is While $(\mathcal{R})$ semicomputable, and hence is also While ${ }^{\mathrm{OR}}(\mathcal{R})$ semicomputable. So the projection of its domain off the $y$ axis:

$$
g\left(x_{1}, x_{2}\right)=_{d f} \exists n: \mathbb{N},\left(x_{1}-2 n\right)^{2}+x_{2}^{2}<1
$$

is projectively While $^{\mathrm{OR}}(\mathcal{R})$ semicomputable.

Figure 5.3: Domain of $g\left(x_{1}, x_{2}\right)$.

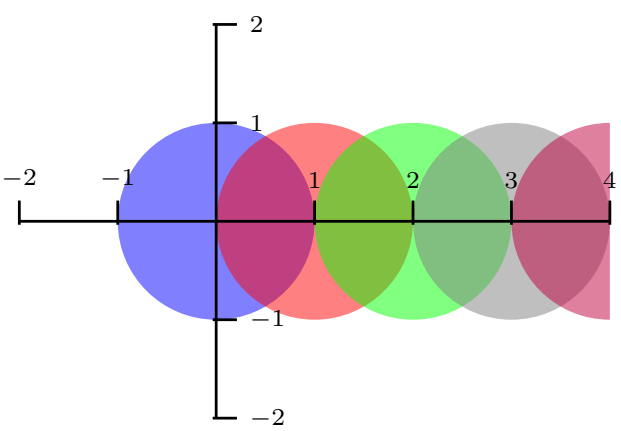

The domain of $g$ is partially pictured in Figure 5.3, again for $x_{1} \leq 4$.

Now consider whether the domain of $g$ is $\boldsymbol{W h i l e}^{\mathrm{OR}}(\mathcal{R})$ semicomputable. By the structure theorem for While $\mathrm{OR}_{(\mathcal{R})}$ semicomputability (Theorem 5.1), that would mean its domain is an effective disjoint union of finite unions of basic sets. In this case, because the domain is connected, it must be a single semialgebraic set. By an analysis of semi-algebraic sets, we can see that this is not the case:

Discussion 5.2.1. Consider the domain of $g\left(x_{1}, x_{2}\right)$. Replace $x_{2}$ by 0.9 , to get the one dimensional set:

$$
\{x \in \mathbb{R} \mid g(x, 0.9)>0\} .
$$

If the domain of $g\left(x_{1}, x_{2}\right)$ is semi-algebraic, then this set should be semialgebraic. 
However, this set is consists of infinitely many intervals (the intervals on which the horizontal line at $x_{2}=0.9$ intersects the "top" of the discs), and therefore is not semi-algebraic. ${ }^{1}$

So $g$ is not semialgebraic, and therefore is not $W_{h i l e} \mathrm{OR}_{(\mathcal{R})}$ semicomputable. Therefore, we have:

Theorem 5. For subsets of $\mathbb{R}^{2}$,

$$
\text { While }^{\mathrm{OR}}(\mathcal{R}) s / \text { comp } \underset{\text { proj-While }}{\mathrm{OR}}(\mathcal{R}) s / \text { comp }
$$

Proof. The ' $\Longrightarrow$ ' direction is clear from the definition of projective While $^{\mathrm{OR}}(\mathcal{R})$ semicomputability. The ' $\Longleftarrow$ ' direction is clear from the above.

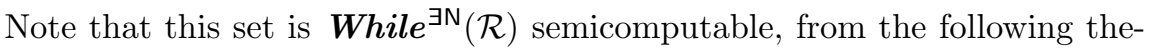
orem given in $[\mathrm{Fu} 14, \S \S 4.6]$ :

Theorem (Structure Theorem for While ${ }^{\exists \mathrm{N}}(\mathcal{R})$ ). For subsets of $\mathbb{R}^{2}$,

$$
\begin{aligned}
\text { While }^{\exists \mathrm{N}}(\mathcal{R}) s / \text { comp } \Longleftrightarrow & \text { countable union of an effective sequence of } \\
& \text { basic sets. }
\end{aligned}
$$

Hence we can also compare While $^{\mathrm{OR}}(\mathcal{R})$ and $\boldsymbol{W h i l e}^{\exists \mathrm{N}}(\mathcal{R})$ semicomputable sets:

Theorem 6. For subsets of $\mathbb{R}^{2}$,

$$
\text { While }^{\mathrm{OR}}(\mathcal{R}) s / \text { comp } \rightleftarrows \text { While }^{\exists \mathrm{N}}(\mathcal{R}) s / \text { comp }
$$

\subsection{Equivalence of projective $W h i l e(\mathcal{R})$ and While $^{\exists \mathrm{N}}(\mathcal{R})$ semicomputability}

In $[$ XFZ15, $\S \S 5.6]$, it was shown that While $^{\exists \mathrm{N}}(\mathcal{R})$ semicomputability was equivalent to projective While $^{\exists \mathrm{N}}(\mathcal{R})$ semicomputability:

\footnotetext{
${ }^{1}$ Thanks again to Prof. Bröcker for pointing this out.
} 
Lemma 5.3.1. For subsets of $\mathbb{R}^{2}$,

$$
\operatorname{proj}_{-} \text {While }^{\exists \mathrm{N}}(\mathcal{R}) s / \text { comp } \Longleftrightarrow \boldsymbol{W h i l e}^{\exists \mathrm{N}}(\mathcal{R}) s / \text { comp }
$$

Essentially, showing this involves replacing the projected arguments with auxiliary variables of sort nat which are existentially quantified over. Because of the continuity of While $^{\exists \mathrm{N}}$ programs, quantifying over naturals suffices for replacing real projected arguments.

We will show that further, While ${ }^{\exists \mathrm{N}}(\mathcal{R})$ semicomputability is equivalent to projective $\boldsymbol{W h i l e}(\mathcal{R})$ semicomputability.

Lemma 5.3.2. For subsets of $\mathbb{R}^{2}$,

$$
\operatorname{proj-While}(\mathcal{R}) s / \text { comp } \Longleftrightarrow \boldsymbol{W h i l e}^{\exists \mathrm{N}}(\mathcal{R}) s / \text { comp }
$$

We need the following fact that follows definitions of the projective models:

Corollary 5.3.3. For subsets of $\mathbb{R}^{2}$,

$$
\begin{aligned}
\operatorname{proj-While}(\mathcal{R}) s / \operatorname{comp} & \Longrightarrow \operatorname{proj}-\boldsymbol{W h i l e}^{\mathrm{OR}}(\mathcal{R}) s / \text { comp } \\
& \Longrightarrow \operatorname{proj} \text {-While }^{\exists \mathrm{N}}(\mathcal{R}) s / \text { comp }
\end{aligned}
$$

Proof. Follows from Theorem 4, Theorem 6 and the definition of the projective models.

Remark 5.3.4. During the proof, we use the While* language in place of the While language for simplicity. We require projection off an arbitrary but finite list of naturals. We can easily represent such a list using a single natural number argument for a While program, but to avoid tediousness we use the While* language for the proof.

Proof of Lemma 5.3.2. The ' $\Longrightarrow$ ' direction follows from Lemma 5.3 .1 and Corollary 5.3.3. 
For the ' $\Longleftarrow$ ' direction, consider any While $^{\exists \mathrm{N}}(\mathcal{R})$ semicomputable set which is the halting set of a While $^{\exists N}$ progrem $P: \mathbb{R}^{2} .^{2}$

We will construct a While $^{*}$ program $P_{0}: \mathbb{R}^{2} \times \mathbb{N}^{*}$ such that the projection of the domain of $P_{0}$ off of $\mathbb{N}^{*}$ is equal to the domain of $P$.

We construct $P_{0}$ from $P$ by replacing each line of the form:

$$
\mathrm{x}^{\mathrm{B}}:=\exists \mathrm{z} P(t, \mathrm{z})
$$

by the two lines:

$$
\begin{gathered}
\mathrm{x}^{\mathrm{B}}:=P(t, \mathbf{z}[\mathrm{i}]) \\
\mathrm{i}:=\mathrm{i}+1
\end{gathered}
$$

where $i$ is a new auxiliary variable which is initialized to 0 at the start of the program.

Then suppose that for some input values $r_{1}, r_{2}: \mathbb{R}^{2}, P\left(r_{1}, r_{2}\right)$ halts. Then since $P$ halted in finitely many steps, there exists a finite list of natural numbers $z_{1}, \ldots, z_{n}$ which are existentially quantified corresponding to the ' $\mathrm{x}^{b}:=\exists \mathrm{z} P(t, \mathrm{z})$ ' lines. This gives an array of naturals $z$ such that $P_{0}\left(r_{1}, r_{2}, z\right)$ halts is given ${ }^{3}$. So the set While $^{\exists N}$ semicomputed by $P$ is seen to also be semicomputable by a projective $\boldsymbol{W h i l e}^{*}$ program and hence a While program (see Remark 5.3.4).

\subsection{Classes of sets semicomputable by models based on the While language}

We now consider the classes of sets semicomputable by the While, While OR $^{\text {W }}$ and $\boldsymbol{W h i l e}^{\exists \mathrm{N}}$ languages and their projective versions.

\footnotetext{
${ }^{2}$ As with all of our (main) results, we restrict our attention to functions on $\mathbb{R}^{2}$. Note that this proof in particular is easily extendable to functions of any type.

${ }^{3}$ Note that the order of the naturals used in the existential quantification steps may have little relation to the order of the $\mathrm{x}^{b}:=\exists z \mathrm{P}(\mathrm{t}, \mathrm{z})$ lines in the code, due to loops and branches.
} 
We begin by combining the results discussed in the previous section:

Theorem 7. For subsets of $\mathbb{R}^{2}$,

$$
\begin{aligned}
\operatorname{proj}_{\text {-While }(\mathcal{R}) s / c o m p} & \Longleftrightarrow p r o j \text {-While }{ }^{\mathrm{OR}}(\mathcal{R}) s / c o m p \\
& \Longleftrightarrow p r o j-\text { While }^{\exists \mathrm{N}}(\mathcal{R}) s / c o m p \\
& \Longleftrightarrow \text { While }^{\exists \mathrm{N}}(\mathcal{R}) s / \text { comp }
\end{aligned}
$$

Proof. Follows from Lemma 5.3.1, Lemma 5.3.2 and Corollary 5.3.3.

We have thus established the existence of three distinct classes of subsets of $\mathbb{R}^{2}$, as shown in the following diagram:
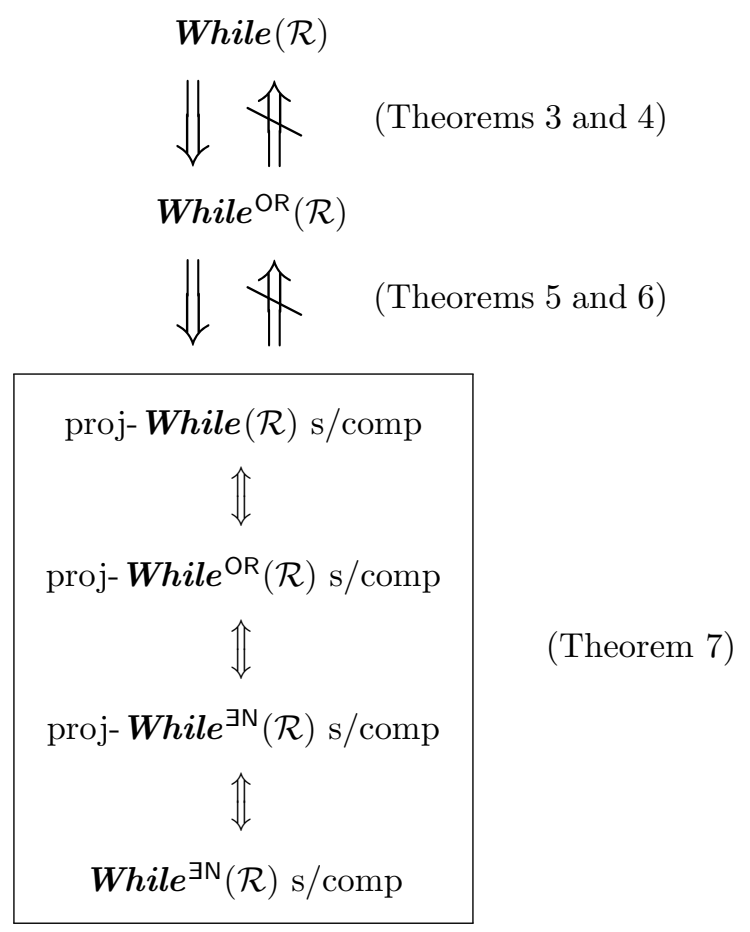

We further have the equivalence of each model in the above diagram with its respective starred version (as shown in Appendix A). 


\section{Chapter 6}

\section{Conclusion and future work}

\subsection{Conclusion}

In this thesis, we investigated the generalisation of two results from classical computability theory to the context of topological algebra of the reals: closure of semicomputable sets under union and the equivalence semicomputable sets of projectively (semi)computable sets. Both results were shown to not hold in the context of the reals (Theorem 2 and Theorem 3 respectively).

In the process we also developed a Structure Theorem for While $(\mathcal{R})$ semicomputability (Theorem 1), and distinguished the classes of sets semicomputed by $\boldsymbol{W h i l e}(\mathcal{R})$, While $^{\mathrm{OR}}(\mathcal{R})$ and $\boldsymbol{W h i l e} \boldsymbol{J N}^{\exists \mathrm{R}}(\mathcal{R})$ programs and their projective versions (section 5.4).

In Appendix B, we show that another result from classical computability theory, Post's Theorem, holds trivially in the case of $\mathcal{R}$, but does not hold more generally for partial algebras. 


\subsection{Future work}

We have compared various classes of subsets of $\mathbb{R}^{2}$ by abstract models based on the While language (section 5.4). Similarly, we would like to investigate concrete models of computability (section 1.1), and compare them amongst themselves and with abstract models. In [TZ04, TZ05], an equivalence was found between certain abstract and concrete models. In [Fu14], several such concrete models were shown to be equivalent.

An open problem in this area is the relationship of models considered in the above papers with Weihrauch's TTE (type two effective) model of computation [Wei00].

The investigation of this problem is intended for a major part of the writer's PhD thesis. 


\section{Bibliography}

[ABR96] C. Andradas, L. Bröcker, and J. Ruiz. Constructible Sets in Real Geometry. Springer, 1996.

[Chu36] A. Church. An unsolvable problem of elementary number theory. —amer-Journal of Mathematical-, 58:345-363, 1936.

[Eng68] E. Engeler. Formal Languages: Automata and Structures. Markham, 1968.

[Fu14] Ming Quan Fu. Characterizations of Semicomputable Sets, and Computable Partial Functions, on the Real Plane. PhD Thesis, Department of Computing \& Software, McMaster University, 2014. Archived in DSpace at http://hdl.handle.net/11375/16066.

[Grz55] A. Grzegorczyk. Computable functions. Fundamenta Mathematicae, 42:168-202, 1955.

[Grz57] A. Grzegorczyk. On the defintions of computable real continuous functions. Fundamenta Mathematicae, 44:61-71, 1957.

[Kle36] S.C. Kleene. General recursive functions of natural numbers. Mathematische Annalen, 112:727-742, 1936.

[Kle52] S.C. Kleene. Introduction to Metamathematics. North Holland, 1952. 
[Tur36] A.M. Turing. On computable numbers, with an application to the Entscheidungsproblem. Proceedings of the London Mathematical Society, 42:230-265, 1936. With correction, ibid., 43, 544-546, 1937. Reprinted in The Undecidable, M. Davis, ed., Raven Press, 1965.

[TZ99] J.V. Tucker and J.I. Zucker. Computation by 'while' programs on topological partial algebras. Theoretical Computer Science, 219:379420,1999 .

[TZ00] J.V. Tucker and J.I. Zucker. Computable functions and semicomputable sets on many-sorted algebras. In S. Abramsky, D. Gabbay, and T. Maibaum, editors, Handbook of Logic in Computer Science, volume 5, pages 317-523. Oxford University Press, 2000.

[TZ04] J.V. Tucker and J.I. Zucker. Abstract versus concrete computation on metric partial algebras. ACM Transactions on Computational Logic, 5:611-668, 2004.

[TZ05] J.V. Tucker and J.I. Zucker. Computable total functions, algebraic specifications and dynamical systems. Journal of Logic and Algebraic Programming, 62:71-108, 2005.

[TZ15] J.V. Tucker and J.I. Zucker. Generalizing computability theory to abstract algebras. In G. Sommaruga and T. Strahm, editors, Turing's Revolution. The Impact of his Ideas about Computability, page (To appear). Birkhauser/Springer Basel, 2015.

[Wei00] K. Weihrauch. Computable Analysis: An Introduction. Springer, 2000.

[XFZ15] Bo Xie, Ming Quan Fu, and Jeffery Zucker. Characterizations of semicomputable sets of real numbers. Journal of Logic and Algebraic Programming, 84:124-154, 2015. 


\section{Appendix A}

\section{The equivalence of}

\section{$\operatorname{While}(\mathcal{R})$ and While $^{*}(\mathcal{R})$}

We wish to justify our claims that the $\boldsymbol{W h i l e}(\mathcal{R})$ and $\boldsymbol{W h i l e}(\mathcal{R})$ are equivalent in terms of computing power.

A similar result was shown in $[\mathrm{TZ00}, \S 4]$ for a total algebra of the reals, by showing that:

(1) the total algebra of the reals has the "term evaluation property" (defined below)

(2) for any $\mathbb{N}$-standard total algebra $A$ with the term evaluation property, a universal While procedure may be constructed for While*

(3) hence, for any $\mathbb{N}$-standard total algebra $A$ with the term evaluation property, While $(A)=$ While $^{*}(A)$.

We may use the same process to show that $\operatorname{While}(\mathcal{R})=\operatorname{While}^{*}(\mathcal{R})$ (and similarly for $\boldsymbol{W h i l e}^{\mathrm{OR}}$ and $\boldsymbol{W h i l e}^{\exists \mathrm{N}}$ ). Steps (2) and (3) can be easily inferred from the respective proofs in $[\mathrm{TZ} 00, \S 4]$, as most of the proofs of those facts 
involve primitive recursive operations on the syntax of the While language, and so the partiality of $\mathcal{R}$ is irrelevant. In these proofs, the only step during the proofs that involves semantics is the use of term evaluation to traverse a "computation tree" for the universal While $(A)$ procedure for While* $(A)$ programs. In that step, however, if a term which is evaluated diverges, the While $^{*}(A)$ program being simulated by the universal procedure would diverge as well, and so the universal procedure behaves as expected.

So we proceed with a proof of Step (1), i.e. that $\mathcal{R}$ has the term evaluation property (cf. [TZ00, Example 4.5]). Note that the partiality of $\mathcal{R}$ presents no problems during the proof; it simply means that the term evaluation function must be partial.

For the remainder of this Appendix, let $A$ be any $\mathbb{N}$-standard (possibly partial) algebra, let $u$ and $v$ be product types of $A$, let $\mathrm{x}$ be a $u$-tuple of variables, let $\boldsymbol{T} \boldsymbol{m}_{\mathrm{x}}(\Sigma)$ be the set of all $\Sigma$-terms with variables among $\mathrm{x}$ only, and for all sorts $s$ or $\Sigma$, let $\boldsymbol{T m}_{\mathrm{x}, s}(\Sigma)$ be the class of such terms of sort $s$.

We define this term evaluation function on $A$ relative to $\mathrm{x}$

$$
T E_{\mathrm{x}, s}^{A}: T m_{\mathrm{x}, s} \times \operatorname{State}(A) \rightarrow A_{s}
$$

by

$$
\boldsymbol{T} \boldsymbol{E}_{\mathrm{x}, s}^{A}(t, \sigma) \simeq \llbracket t \rrbracket^{A} \sigma .
$$

We represent the term evaluation function on $A$ on relative to $\mathrm{x}$ by the function

$$
\boldsymbol{t} \boldsymbol{e}_{\mathrm{x}, s}^{A}:\left\ulcorner\boldsymbol{T} \boldsymbol{m}_{\mathrm{x}, s}\right\urcorner \times A^{u} \rightarrow A_{s}
$$

defined by

$$
t e_{\mathrm{x}, s}^{A}(\ulcorner t\urcorner, a) \simeq \llbracket t \rrbracket^{A} \sigma,
$$

where $\sigma$ is any state on $A$ such that $\sigma[\mathrm{x}]=a$ (this is well defined, by Lemma 3.4 from [TZ00]). 
Definition A.0.1. An algebra $A$ has the term evaluation property $(T E P)$ if for all $\mathrm{x}$ and $s, \boldsymbol{t} \boldsymbol{e}_{\mathrm{x}, s}^{A}$ is $\boldsymbol{W h i l e}(A)$ computable.

Lemma A.0.2. $\mathcal{R}$ has the TEP.

Proof (outline).

We may give the following primitive recursive algorithm which shows the computability of $\boldsymbol{t} \boldsymbol{e}_{\mathrm{x}, s}^{\mathcal{R}}$; for instance, in the case that $s \equiv$ bool:

$$
\begin{aligned}
& \boldsymbol{t} \boldsymbol{e}_{\mathrm{x}, \text { bool }}^{\mathcal{R}}\left(\left\ulcorner t_{s} \text { comp } r_{s}\right\urcorner, a\right) \simeq \boldsymbol{t} \boldsymbol{e}_{\mathrm{x}, s}^{\mathcal{R}}\left(\left\ulcorner t_{s}\right\urcorner, a\right) \text { comp } \boldsymbol{t} \boldsymbol{e}_{\mathrm{x}, s}^{\mathcal{R}}\left(\left\ulcorner r_{s}\right\urcorner, a\right) \\
& \boldsymbol{t} \boldsymbol{e}_{\mathrm{x}, \text { bool }}^{\mathcal{R}}\left(\left\ulcorner\operatorname{not}^{\mathrm{B}}\left(b_{1}\right)\right\urcorner, a\right) \simeq \operatorname{not}^{\mathrm{B}}\left(\boldsymbol{t} \boldsymbol{e}_{\mathrm{x}, \text { bool }}^{\mathcal{R}}\left(\left\ulcorner b_{1}\right\urcorner, a\right)\right) \\
& \boldsymbol{t} \boldsymbol{e}_{\mathrm{x}, \text { bool }}^{\mathcal{R}}\left(\left\ulcorner b_{1} \text { op } b_{2}\right\urcorner, a\right) \simeq \boldsymbol{t} \boldsymbol{e}_{\mathrm{x}, \text { bool }}^{\mathcal{R}}\left(\left\ulcorner b_{1}\right\urcorner, a\right) \text { op } \boldsymbol{t} \boldsymbol{e}_{\mathrm{x}, \text { bool }}^{\mathcal{R}}\left(\left\ulcorner b_{2}\right\urcorner, a\right)
\end{aligned}
$$

where sort $s$ is either nat or real, $b_{1}$ and $b_{2}$ are terms of sort bool, comp is a comparison operator on $s$ (one of $\mathrm{eq}^{\mathrm{N}}$, less ${ }^{\mathrm{N}}, \mathrm{eq}^{\mathrm{R}}$ or less ${ }^{\mathrm{R}}$ ), and op is a binary boolean operator.

We omit the corresponding definitions for the cases $s \equiv$ real and $s \equiv$ nat.

The equivalence of $\boldsymbol{W h i l e}(\mathcal{R})$ and $\boldsymbol{W h i l e}^{*}(\mathcal{R})$ now follows from the above Lemma and the preceeding discussion. 


\section{Appendix B}

\section{A counterexample to Post's}

\section{Theorem for partial}

\section{algebras}

For total standard algebras, we have the following theorem [TZ00, $\S 5.2]$, [TZ15, $\S \S 6.1]$ :

Theorem 8 (Post's theorem for While semicomputability on total algebras). For any relation $R$ on any total algebra $A$,

$R$ is While $(A)$ computable $\Longleftrightarrow R$ and $R^{c}$ are While $(A)$ semicomputable.

We will show that Post's Theorem also holds on the partial algebra $\mathcal{R}$, but that there are some partial algebras on which it does not hold.

Theorem 9 (Post's theorem for While $(\mathcal{R})$ semicomputability). For any relation $R$ on $\mathcal{R}$,

$R$ is While $(\mathcal{R})$ computable $\Longleftrightarrow R$ and $R^{c}$ are While $(\mathcal{R})$ semicomputable. 
Proof. The ' $\Longrightarrow$ ' direction is obvious; if $R$ is computable, then $R^{c}$ is also computable and hence semicomputable.

For the ' $\Longleftarrow$ ' direction, recall that by the Structure Theorem for While $(\mathcal{R})$, if $R$ and $R^{c}$ are $\boldsymbol{W h i l e}(\mathcal{R})$ semicomputable then they are unions of effective disjoint sequences of basic sets, and recall that those basic sets are open. Then since any union of open sets is open, both $R$ and $R^{c}$ are open, and since the complement of an open set is closed, they are in fact both clopen. Then since the only clopen sets of the reals are the empty set and $\mathbb{R}^{2}, R$ is one of those two, and is clearly computable.

We now give an example of a standard partial algebra ${ }^{1} \mathcal{D}$ such that Post's theorem does not hold for its While semicomputable subsets.

Define a signature $\Sigma^{\mathcal{D}}$ :

$\begin{array}{ll}\text { signature } & \Sigma^{\mathcal{D}} \\ \text { sorts } & \text { data, bool } \\ \text { functions } & 0: \rightarrow \text { data } \\ & F, G: \text { data } \rightarrow \text { data } \\ & \text { eq }: \text { data }^{2} \rightarrow \text { bool }\end{array}$

and algebra $\mathcal{D}$ :

\begin{tabular}{|ll|}
\hline algebra & $\mathcal{D}$ \\
carriers & $\mathbb{D}, \mathbb{B}$ \\
functions & $0: \rightarrow \mathbb{D}$ \\
& $\mathrm{F}^{\mathrm{D}}, \mathrm{G}^{\mathrm{D}}: \mathbb{D} \rightarrow \mathbb{D}$ \\
& $=: \mathbb{D}^{2} \rightarrow \mathbb{B}$ \\
\hline
\end{tabular}

where $\mathbb{D}=\{a, b, 0\}$ is the carrier for sort data and $\mathrm{F}^{\mathrm{D}}$ and $\mathrm{G}^{\mathrm{D}}$ are defined as follows:

\footnotetext{
${ }^{1}$ The proof for an $\mathbb{N}$-standard algebra is a routine, but tedious, extension. To see this observe that simply adding the naturals to the algebra gives us no additional computing power with regards to the data sort.
} 


$$
\begin{aligned}
& \mathrm{F}^{\mathrm{D}}(x)= \begin{cases}x & \text { if } x=a \\
\uparrow & \mathrm{o} / \mathrm{w}\end{cases} \\
& \mathrm{G}^{\mathrm{D}}(x)= \begin{cases}x & \text { if } x \neq a \\
\uparrow & \mathrm{o} / \mathrm{w}\end{cases}
\end{aligned}
$$

and $={ }^{\mathrm{D}}$ is the equality operator for sort $\mathbb{D}$.

Remark B.0.1. Any composition of the $\mathrm{F}^{\mathrm{D}}$ and $\mathrm{G}^{\mathrm{D}}$ functions is eliminable, in that for any term $t$ :

$$
\text { - } \mathrm{F}^{\mathrm{D}}\left(\mathrm{F}^{\mathrm{D}}(t)\right) \simeq \mathrm{F}^{\mathrm{D}}(t)
$$

- $\mathrm{G}^{\mathrm{D}}\left(\mathrm{G}^{\mathrm{D}}(t)\right) \simeq \mathrm{G}^{\mathrm{D}}(t)$

- $\mathrm{F}^{\mathrm{D}}\left(\mathrm{G}^{\mathrm{D}}(t)\right) \uparrow$

- $\mathrm{G}^{\mathrm{D}}\left(\mathrm{F}^{\mathrm{D}}(t)\right) \uparrow$.

We will prove:

Proposition B.0.2. Any boolean valued While $\left(\Sigma^{\mathcal{D}}\right)$ program which halts on inputs $a$ and $b$ must give the same output in both cases. $^{2}$

Remark B.0.3. It follows from Proposition B.0.2 that $\{a\}$ and $\{a\}^{c}=\{b, 0\}$ are both clearly semicomputable, but neither is computable.

In preparation for the proof of Proposition B.0.2, we need two related notions: compatible set of states and set of distinguished variables.

Definition B.0.4 (Compatible states; Distinguished variables).

Let $\underline{A}$ be a $\Sigma$-structure and $s$ a $\Sigma$-sort. Suppose for some $n \geq 2$, we have:

- $n$ states $\sigma_{1}, \ldots, \sigma_{n}$,

\footnotetext{
${ }^{2}$ It is certainly possible for a data valued program to give different outputs on $a$ and $b$; consider the identity function.
} 
- $n$ distinct elements $a_{1}, \ldots, a_{n}$ of $A_{s}$ and

- a finite set $V$ of variables of sort $s$, such that

$$
\forall \mathrm{x} \notin V, \sigma_{1}(\mathrm{x})=\ldots=\sigma_{n}(\mathrm{x})
$$

and

$$
\forall \mathrm{x} \in V, \sigma_{1}(\mathrm{x})=a_{1}, \ldots, \sigma_{n}(\mathrm{x})=a_{n}
$$

We call $\left\{\sigma_{1}, \ldots, \sigma_{n}\right\}$ a compatible set of states with respect to $V$.

We call $V$ the set of distinguished variables for these states.

Note that the set of distinguished variables is unique for any particular set of compatible states.

Now (returning to the special case of $\underline{A}=\mathcal{D}, s=$ data) take two compatible states $\sigma_{a}$ and $\sigma_{b}$ over $\mathcal{D}$ with some set of distinguished variables $V$ such that for all $\mathrm{v} \in V$

$$
\sigma_{a}(\mathrm{v})=a, \quad \sigma_{b}(\mathrm{v})=b .^{3}
$$

For the remainder of the section, we let $S$ be any statement in $\boldsymbol{W h i l e}\left(\Sigma^{\mathcal{D}}\right)$ for which the following assumption holds:

Assumption B.0.5. S halts on $\sigma_{a}$ and $\sigma_{b}$.

In order to state and prove Lemma B.0.6 below, from which Proposition B.0.2 will follow, we need to reason about the snapshot sequences (recall the definition in section 2.9) generated by $S$ at $\sigma_{a}$ and $\sigma_{b}$.

We write:

$$
\begin{aligned}
& \left(\boldsymbol{\sigma}_{a}, \boldsymbol{S}\right)=\left(\sigma_{a}^{0}, S_{a}^{0}\right),\left(\sigma_{a}^{1}, S_{a}^{1}\right),\left(\sigma_{a}^{2}, S_{a}^{2}\right), \ldots \\
& \left(\boldsymbol{\sigma}_{b}, \boldsymbol{S}\right)=\left(\sigma_{b}^{0}, S_{b}^{0}\right),\left(\sigma_{b}^{1}, S_{b}^{1}\right),\left(\sigma_{b}^{2}, S_{b}^{2}\right), \ldots
\end{aligned}
$$

\footnotetext{
${ }^{3}$ For instance, the states which assign $a$ and $b$ respectively to all $\mathrm{v} \in V$ and 0 to every other variable are good candidates for $\sigma_{a}$ and $\sigma_{b}$.
} 
We may now state Lemma B.0.6:

Lemma B.0.6. The snapshot sequences $\left(\boldsymbol{\sigma}_{a}, \boldsymbol{S}\right)$, and $\left(\boldsymbol{\sigma}_{b}, \boldsymbol{S}\right)$ are isomorphic in that for all $n, S_{a}^{n} \equiv S_{b}^{n}$, and $\sigma_{a}^{n}$ and $\sigma_{b}^{n}$ are compatible with a set $V^{n}$ of distinguished variables which are assigned $a$ and $b$ when the starting states are $\sigma_{a}$ and $\sigma_{b}$ respectively.

In fact, the set $V^{n}$ can be effectively identified given any $\sigma_{a}, \sigma_{b}$ and $n$.

Lemma B.0.6 says, in effect, that the computations of $S$ on $\sigma_{a}$ and $\sigma_{b}$ are "essentially the same". From this, Proposition B.0.2 follows.

The following Remark is central to the induction step of the proof of Lemma B.0.6:

Remark B.0.7. Any subterm of a term which is evaluated at stage $n$ of the computation of $S$ at $\sigma_{a}$ or $\sigma_{b}$ cannot have the form $\mathrm{F}(\mathrm{y})$ or $\mathrm{G}(\mathrm{y})$ where $\mathrm{y}$ is a distinguished variable of the states $\sigma_{a}^{n}$ and $\sigma_{b}^{n}$, since that would violate Assumption B.0.5. ${ }^{4,5}$ This essentially restricts our tests on the input variable to boolean combinations of tests for equality; specifically, due to the lack of closed terms for $a$ and $b$, equality between variables or tests for zero.

The fact that tests on the input are limited to tests for equality between variables and tests for zero will allow us to show that, restricting our attention to states for which all variables of sort data are assigned the same values (such as $\sigma_{a}$ and $\sigma_{b}$ ) and statements $S$ for which Assumption B.0.5 holds, branching decisions on input states which only vary on their data values are the same between those states, and can be trivially predetermined.

We now prove Lemma B.0.6 by giving an algorithm to find the compatible variable set for $\sigma_{a}^{n}$ and $\sigma_{b}^{n}$ for any $n$.

\footnotetext{
${ }^{4}$ Recall by Remark B.0.1 that we can eliminate occurrences of $F$ and $G$ from other contexts in $S$.

${ }^{5}$ The existance of compatible states and distinguished variables at stage $n$ is given by Lemma B.0.6
} 
Proof of Lemma B.o.6. By course of values induction on the computation length of $S$ at $\sigma_{a}{ }^{6}$

Assume that for $n$, Lemma B.0.6 holds for any $S$ such that

$\operatorname{CompLen}^{D}\left(S, \sigma_{a}\right)<n$. We show that it also holds for $S$ such that

$\operatorname{CompLen}^{D}\left(S, \sigma_{a}\right)=n$. There are 5 cases to consider based on the form of $S$ :

(1) $S$ 三 skip : Trivial (compare with (2)).

(2) $S \equiv \mathrm{x}:=t$ : By $\operatorname{CompLen}^{D}\left(S, \sigma_{a}\right)=n$, we have $n=1$, and so

$\operatorname{Comp}^{D}(S, \sigma, n)=\langle|S|\rangle^{D}=\sigma\left\{\mathrm{x} / \llbracket t \rrbracket^{D} \sigma\right\} .^{7}$ Consider sub-cases based on $t:^{8}$

(i) $t \equiv 0:$ Then take $V^{\prime}=V \backslash\{\mathrm{x}\}$, and observe that $V^{\prime}$ is an appropriate set of distinguished variables.

(ii) $t \equiv \mathrm{y}:$ Then if $\mathrm{y} \in V$, take $V^{\prime}=V \cup\{\mathrm{x}\}$, and otherwise take $V^{\prime}=V$. Then observe that $V^{\prime}$ is an appropriate set of distinguished variables .

(iii) $t \equiv \mathrm{F}^{\mathrm{D}}(\mathrm{y})$ where $\mathrm{y}$ is not a distinguished variable of $\sigma_{a}, \sigma_{b}$, and $\sigma_{c}$ : then take $V^{\prime}=V \backslash\{\mathrm{x}\}$, and observe that $V^{\prime}$ is an appropriate set of distinguished variables.

(iv) $t \equiv \mathrm{G}^{\mathrm{D}}(\mathrm{y})$ where $\mathrm{y}$ is not a distinguished variable of $\sigma_{a}, \sigma_{b}$, and $\sigma_{c}$ : similar to (iii).

(v) $t \equiv \mathrm{F}^{\mathrm{D}}(\mathrm{y})$ where $\mathrm{y}$ is a distinguished variable of $\sigma_{a}$ : by Remark B.0.7, such a term cannot appear in $S$.

(vi) $t \equiv \mathrm{G}^{\mathrm{D}}(\mathrm{y})$ where $\mathrm{y}$ is a distinguished variable of $\sigma_{a}:$ similar to (v).

(vii) $t \equiv \mathrm{F}^{\mathrm{D}}(0)$ : by Assumption B.0.5, since $\mathrm{F}_{a}^{\mathrm{D}}(0)$ diverges at all states, such a term cannot appear in $S$.

(viii) $t \equiv \mathrm{G}^{\mathrm{D}}(0):$ similar to (vii).

\footnotetext{
${ }^{6}$ It will follow from the proof that the computation length of $S$ at $\sigma_{a}$ is the same as the computation length of $S$ at $\sigma_{b}$.

${ }^{7}$ By the definition of $\boldsymbol{C o m p}^{D}$ given in [TZ00, $\left.\S \S 3.4\right]$.

${ }^{8}$ Recall by Remark B.0.1 that we may limit the use of $F_{a}$ and $F_{b}$ to this finite set of cases.
} 
So for each which can appear in $S$, we have that $\sigma_{a}^{n}$ and $\sigma_{b}^{n}$ are compatible with respect to some $V^{\prime}$. We also clearly have that $S_{a}^{n} \equiv S_{b}^{n} \equiv$ skip.

(3) $S \equiv S_{1} ; S_{2}$ : Let $\boldsymbol{C o m p L e n}{ }^{D}\left(S_{1}, \sigma_{a}\right)=k<n$ and

$\operatorname{CompLen}^{D}\left(S_{2}, \sigma_{a}^{k}\right)=l=n-k$. By the induction hypothesis, since $i<n$, $S_{a}^{i} \equiv S_{b}^{i}$ and $\sigma_{a}^{i}$ and $\sigma_{b}^{i}$ are compatible with respect to a set of variables $V_{1}$. Now consider the snapshot sequences $\left(\boldsymbol{\sigma}_{a}^{i}, \boldsymbol{S}_{\boldsymbol{a}}^{\boldsymbol{i}}\right)$ and $\left(\boldsymbol{\sigma}_{\boldsymbol{b}}^{\boldsymbol{i}}, \boldsymbol{S}_{\boldsymbol{b}}^{\boldsymbol{i}}\right)$. By the induction hypothesis, since $j<n, S_{a}^{i j} \equiv S_{b}^{i j}$ and $\sigma_{a}^{i j}$ and $\sigma_{b}^{i j}$ are compatible with respect to a set of variables $V_{2}$. Now note that $S_{a}^{n} \equiv S_{a}^{i j}$ and $S_{b}^{n} \equiv S_{b}^{i j}$, and $\sigma_{a}^{i j}=\sigma_{a}^{n}$ and $\sigma_{b}^{i j}=\sigma_{b}^{n}$. So by taking $V^{\prime}=V_{2}$, we have that $S_{a}^{n} \equiv S_{b}^{n}$ and $\sigma_{a}^{n}$ and $\sigma_{b}^{n}$ are compatible with respect to $V^{\prime}$.

(4) $S \equiv$ if $t^{\mathrm{B}}$ then $S_{1}$ else $S_{2}$ fi : Note that the only boolean test for $A$ is $=$, so $t_{b}$ is a combination of tests of equality between variables or tests for zero ${ }^{9}$. It is easy to see that $\llbracket t_{b} \rrbracket^{D} \sigma_{a}=\llbracket t_{b} \rrbracket^{D} \sigma_{b}{ }^{10}$ Now our induction hypothesis holds for the snapshot sequences $\left(\boldsymbol{\sigma}_{a}, \boldsymbol{S}_{\mathbf{1}}\right),\left(\boldsymbol{\sigma}_{b}, \boldsymbol{S}_{\mathbf{1}}\right),\left(\boldsymbol{\sigma}_{a}, \boldsymbol{S}_{\mathbf{2}}\right)$ and $\left(\boldsymbol{\sigma}_{b}, \boldsymbol{S}_{\mathbf{2}}\right)$. And for the case that $\llbracket t_{b} \rrbracket^{D} \sigma_{a}=$ true, it is clear it also holds for the snapshot sequences

$$
\begin{aligned}
& \left(\boldsymbol{\sigma}_{a}, \boldsymbol{S}\right)=\left(\sigma_{a}, S\right),\left(\boldsymbol{\sigma}_{a}, \boldsymbol{S}_{\mathbf{1}}\right) \\
& \left(\boldsymbol{\sigma}_{b}, \boldsymbol{S}\right)=\left(\sigma_{b}, S\right),\left(\boldsymbol{\sigma}_{b}, \boldsymbol{S}_{\mathbf{1}}\right)
\end{aligned}
$$

The case that $\llbracket t^{\mathrm{B}} \rrbracket^{D} \sigma_{a}=$ false is similar.

(5) $S \equiv$ while $t^{\mathrm{B}}$ do $S_{0}$ od: As in (4), we have that $t_{b}$ is a combination of zero tests or equality between variables, and $\llbracket t_{b} \rrbracket^{D} \sigma_{a}=\llbracket t_{b} \rrbracket^{D} \sigma_{b}$. Now our induction hypothesis holds for the snapshot sequence $\left(\boldsymbol{\sigma}_{a}, \boldsymbol{S}_{\mathbf{0}}\right)$. And for the case $\llbracket t_{b} \rrbracket^{D} \sigma_{a}=$ true, it must also hold for the snapshot sequences

\footnotetext{
${ }^{9}$ See Remark B.0.7.

${ }^{10} \mathrm{By}$ assumption B.0.5, $t_{b}$ does not diverge on any state.
} 


$$
\begin{aligned}
& \left(\boldsymbol{\sigma}_{a}, \boldsymbol{S}\right)=\left(\sigma_{a}, S\right),\left(\boldsymbol{\sigma}_{a}, \boldsymbol{S}_{\mathbf{0}} ; \boldsymbol{S}\right) \\
& \left(\boldsymbol{\sigma}_{b}, \boldsymbol{S}\right)=\left(\sigma_{b}, S\right),\left(\boldsymbol{\sigma}_{b}, \boldsymbol{S}_{\mathbf{0}} ; \boldsymbol{S}\right)
\end{aligned}
$$

because although $S_{0} ; S$ is a more complex program that $S$, by Assumption B.0.5, the while loop must halt and so its computation length must be less, hence we can apply the induction hypothesis.

The case that $\llbracket t_{b} \rrbracket^{D} \sigma_{a}=$ false is similar.

This proves Lemma B.0.6 and hence Proposition B.0.2, thus providing (by Remark B.0.3) a counterexample to Posts Theorem on the algebra $\mathcal{D}$. 